\title{
The Determination of Surface Roughness from Reflected Step Edges
}

\author{
Ronald A. Stone and Steven A. Shafer
}

CMU-RI-TR-93-25

The Robotics Institute

Carnegie Mellon University

Pittsburgh, Pennsylvania 15213

November 1993

Copyright $(191993$ Carnegie Mellon University

This research was sponsored by the Avionics Laboratory, Wright Research and Development Center, Aeronautical Systems Division (AFSC), U.S. Air Force, Wright-Patterson AFB, OH 45433-6543 under Contract F33615-90-C1465, Arpa Order No. 7597. 



\section{Table of Contents}

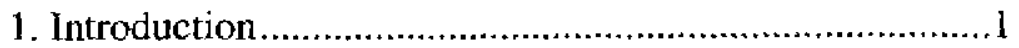

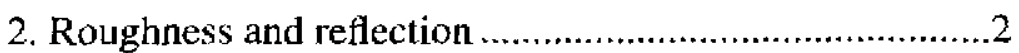

3. One dimensional Gaussian heights ...............................5

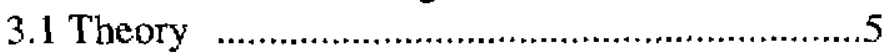

3.2 Experiment ….............................................

4. Other roughness models.............................................24

4.1 Isotropic Gaussian height model....................24

4.2 General one-dimensional FADF …...............25

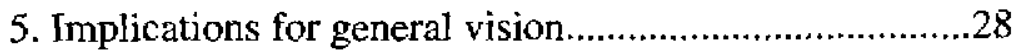

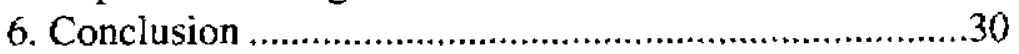

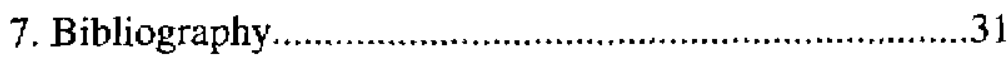

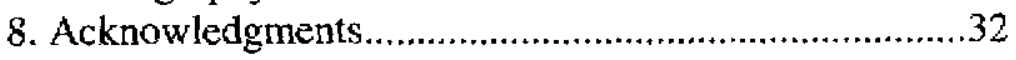

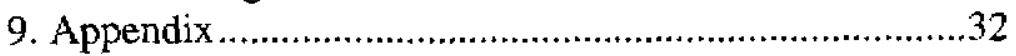





\section{List of Figures}

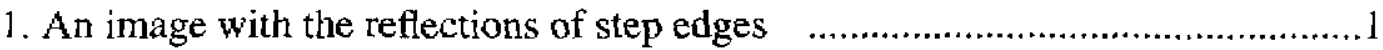

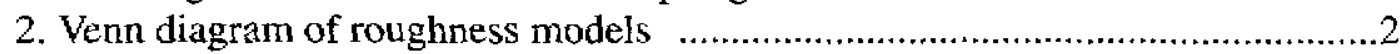

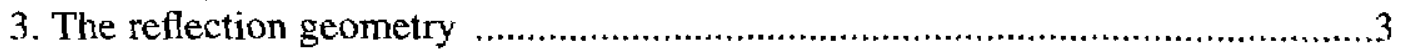

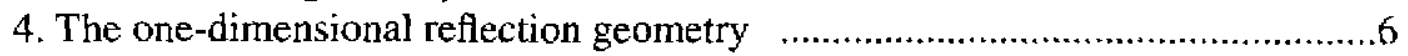

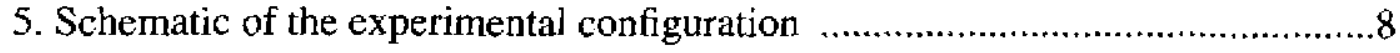

6. The reflection geometry for the experimental configuration ...........................9

7. Images of reflected step edges

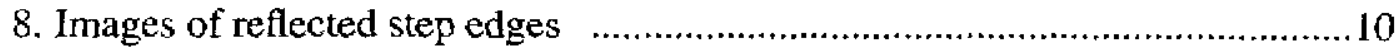

9. Image of a reflected step edge ................................................................ 10

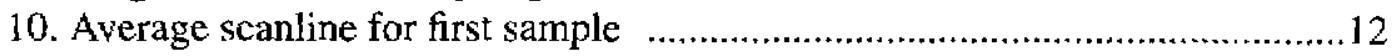

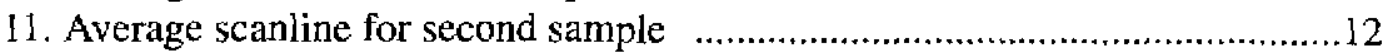

12. Average scanline for third sample …....................................................13

13. Average scanline for fourth sample ......................................................13

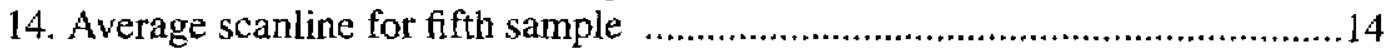

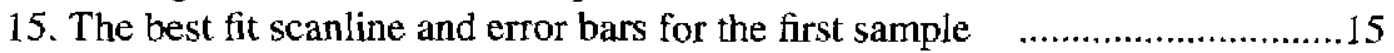

16. The best fit scanline and error bars for the second sample $\quad \ldots \ldots \ldots \ldots \ldots \ldots \ldots . . . \ldots 16$

17. The best fit scanline and error bars for the third sample $\ldots \ldots \ldots \ldots \ldots \ldots \ldots \ldots \ldots . . . . . . .16$

18. The best fit scanline and error bars for the fourth sample $\ldots \ldots \ldots \ldots \ldots \ldots \ldots \ldots . . .17$

19. The best fit scanline and error bats for the fifth sample ...........................17

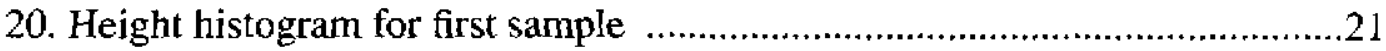

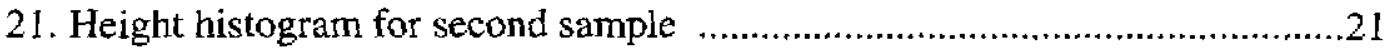

22. Height histogram for third sample ...................................................22

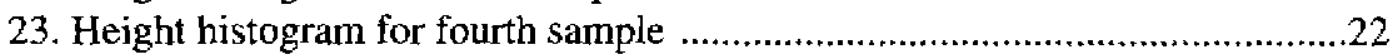

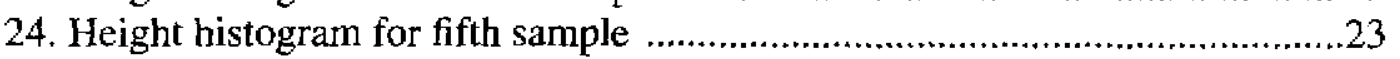

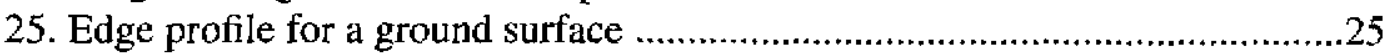

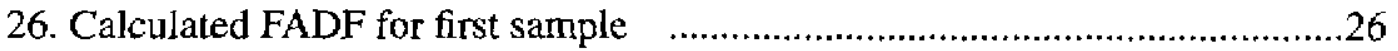

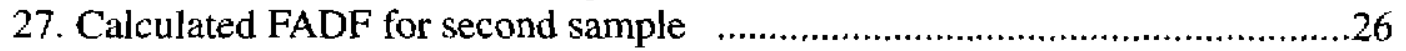

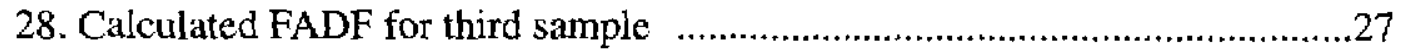

29. Calculated FADF for fourth sample ....................................................27

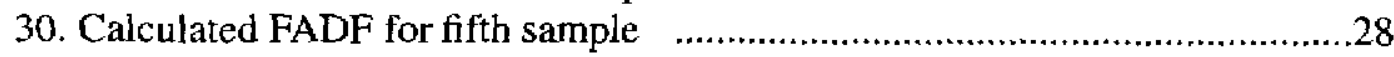

31. Edge profiles for the same surface with different source positions ............29

32. Edge profiles for different surfaces with different source positions .............29

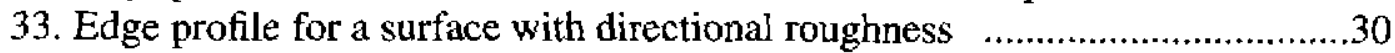

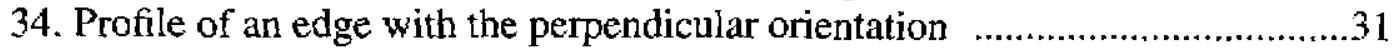





\section{List of Tables}

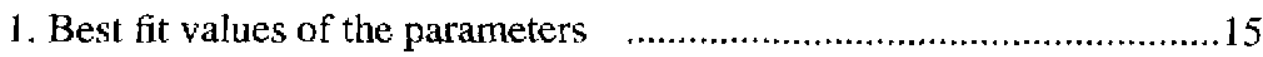

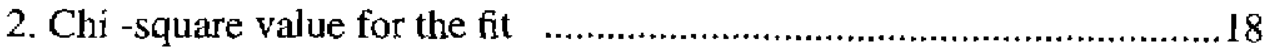

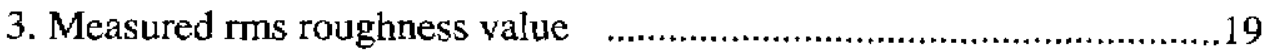

4. Calculated correlation length ......................................................20

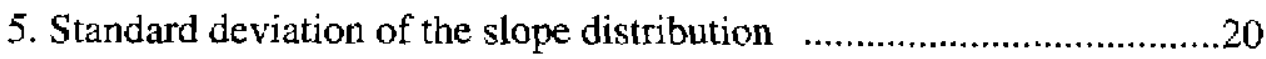

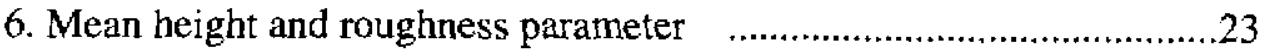





\begin{abstract}
The ability to measure the roughness of surfaces will be important for general purpose machine vision systems, in which a robot attempts to passively gain information about its environment. Although the traditional study of profilometry is well developed, its methods are unsuitable for this endeavor, since they require specialized apparatuses such as laser interferometers and styluses. We propose a method for the visual determination of surface roughness which does not require strong environmental constraints such as coherent light and small samples of the surface in question. We determine surface roughness by the measurement of the sharpness of the edges of an image reflected in a rough surface. We choose step edges since they will be present in many environments, and because the methods of edge detection and localization are mature. For these initial experiments, we assume a common surface roughness model of simple form, which we believe models milled or rolled surfaces. We show that if we also assume a known shape for the reflective suface and a known position for the viewer, we may calculate a roughness parameter. For our surface roughness model, we show that the resulting intensity distribution may be given in closed form. We then show the results of experiments performed with planar surfaces in a controlled environment and give the calculated roughness parameter. We compare these results with the values of the roughness as measured with a stylus profilometer, and find that the results are quite different. We discuss the possibilities that this difference is due to the non-Gaussian character of the surface height distribution, and the possibility that the difference arises from the difference in measurement procedures. We next show some extensions to other type of roughness functions, and discuss some new methods of roughness estimation which may yield information about a broader class of roughness models. Finally, we discuss the additional issues in extending and applying this approach to a general vision system.
\end{abstract}





\section{Introduction}

The determination of surface roughness is important in many areas, from quality control in factories, to general purpose machine vision systems. Although there exist many instruments which measure roughness accurately, such as laser interferometric devices and stylus profilometers, these instruments all require strong constraints on the environment and the samples, for example, the necessity for coherent light or limits on the sample size. These constraints make such devices impractical for a general purpose vision system, in which a robot attempts to gain information about its environment. It is obvious that we can obtain some information about roughness without these kinds of constraints, since humans can often make crude guesses of surface roughness, and can even differentiate surfaces according to roughness, solely on the basis of visual information. For example, technicians in mills sometimes check the roughness of sheet metal rolling equipment by observing the reflection of their fingers in the rollers. As another example, consider Figure 1. This is a picture of a stainless steel ball bearing that has been roughened by sand blasting. In the bearing, we see imperfect images of the objects in the room, including rows of fluorescent lights, windows, pens, and even the photographer. Most people will be able to make a guess at the roughness of the metal, and say that

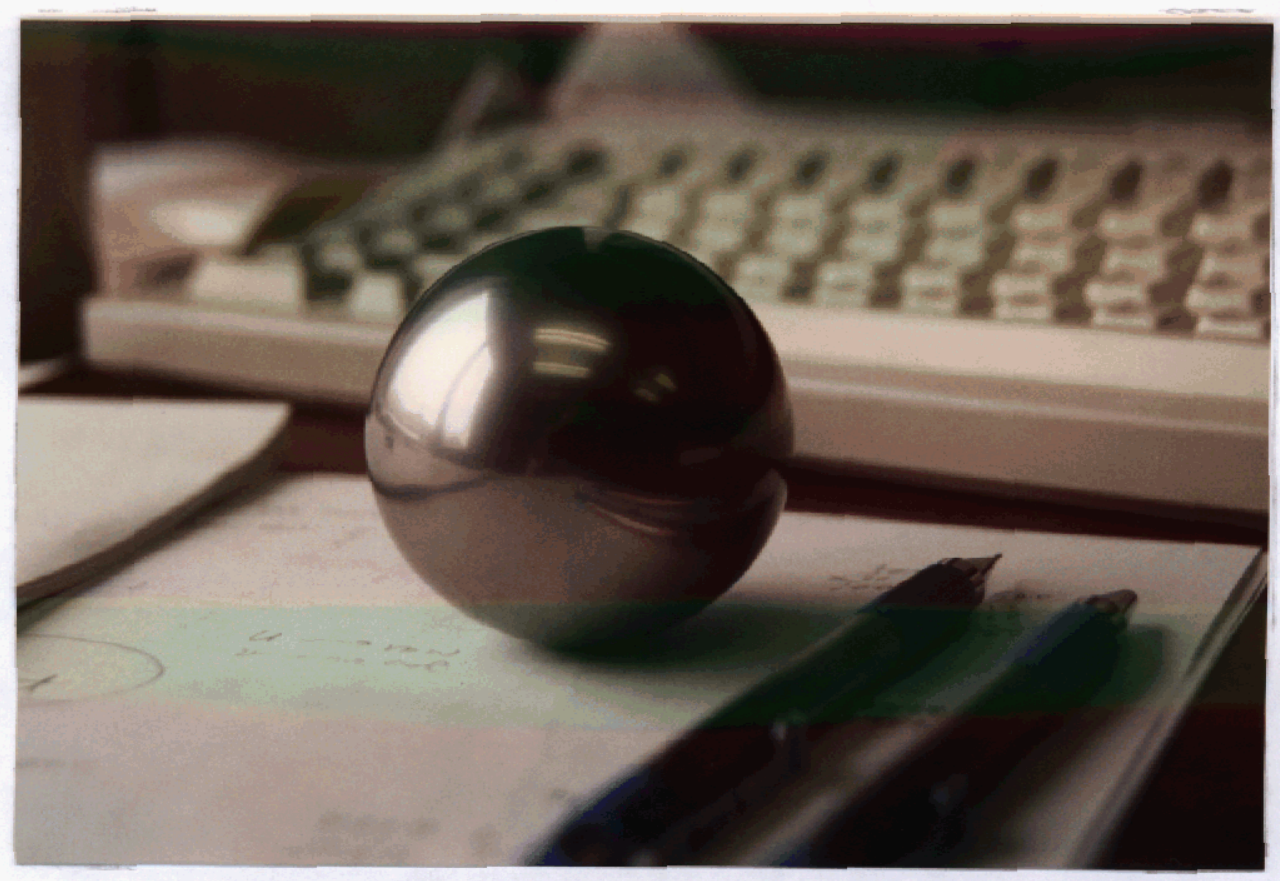

Figure 1: An image with the reflections of step edges

it is "smooth", "a little rough", and so on, even though they lack sophisticated instruments. We believe that they do this by analyzing the blurriness of the reflected image of the environment in the surface. We propose to mimic this ability by analyzing reflected step edges; step edges are valuable for analysis because they provide considerable constraint on the form of the reflected image, are present in almost all environments, and will be relatively easy to find in images, since the field of edge detection is well developed. We will first present a taxonomy of the commonly used surface roughness models. Then, in order to describe reflected step edges, we must choose one of these models, so we use the one which is most common among computer vision researchers, and which models surfaces which have been rolled or milled. We believe this model is general enough to yield useful results. We then find the form of the image of the reflected step edge as a function of roughness, and show the results of our experiments with rough surfaces. 


\section{Roughness and reflection}

Let us begin by describing some of the rough surface models used by machine vision researchers. Figure 2 presents a simple taxonomy of these models; this listing is by no means complete, but clarifies the relationships between some

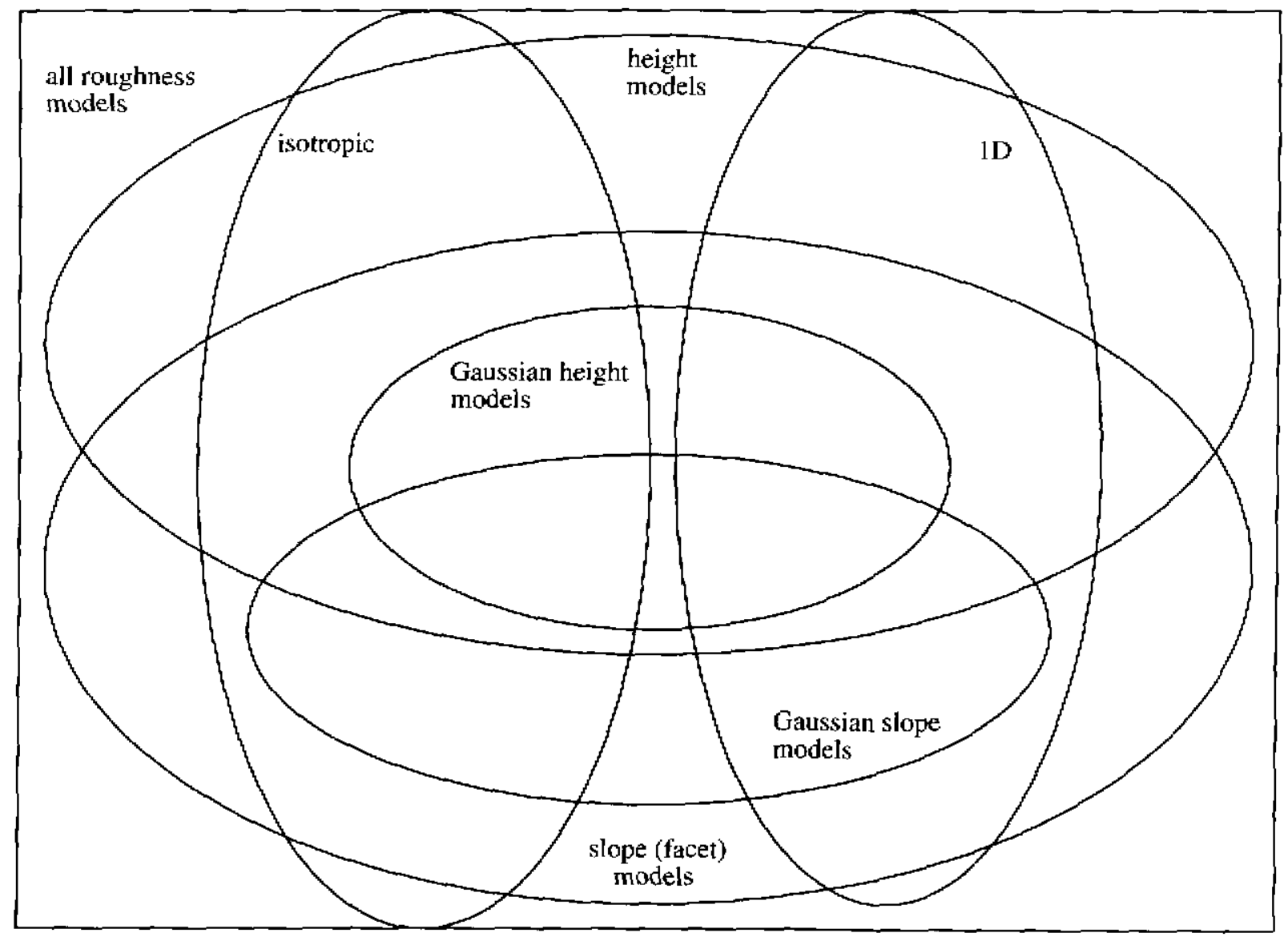

Figure 2: Venn diagram of roughness models

of the commonly used models. We may parametrize a surface by means of its height values or its slopes, and the choice will determine if we want to solve for the reflected light intensity by means of the methods of physical optics or of geometrical optics. The diagram shows that there is some overlap between the two formalisms, because some height-based models have a slope-based interpretation. Next, we may consider the directionality of the roughness, although most machine vision researchers consider only the limiting cases of isotropic and one-dimensional roughness. It is also common to assume that the heights or slopes follow a Gaussian distribution. Note that the Gaussian height models have a slope interpretation, and thus also fall into the category of slope-based models. Not all Gaussian slope models have a height-based interpretation, however. The isotropic and one-dimensional Gaussian models are the most commonly used classes of models. The isotropic Gaussian height model and the one-dimensional Gaussian height model are those proposed by Beckmann and Spizzichino[1]. The isotropic Gaussian slope model is that proposed by Torrance and Sparrow[12], and is probably the most commonly used model in computer vision research $[3,4,5,7,9]$. The one-dimensional Gaussian slope model is the one-dimensional analogue of this model. Note also that the isotropic Gaussian height model and the isotropic Gaussian slope model are equivalent in the limiting case, as are the one-dimensional Gaussian height model and the one-dimensional Gaussian slope model[ $[1,8]$. Thus, these classes overlap. Other models, such as fractal height models, appear in the optics literature[6], but are uncommon in machine vision research, probably due to their complex nature. These models do not appear in the diagram, 
since their relationship to other models is uncertain, although they would belong in the category of height-based models.

We will quantify the scattering from rough surfaces with a slope-based model, in which light reflects from small, mirror-like facets which comprise the surface; we will use the methods of geometrical optics to calculate the reflected radiance distribution. These slope-based models do not deal with the individual facets on the surface, but rather the statistical properties of the set of all facets on the surface.

Let us now derive the radiance of light reflected by a rough faceted surface. This derivation parallels that given by Torrance and Sparrow[12]. Consider a small planar patch of the surface under study, of size dA (see Figure 3 ).

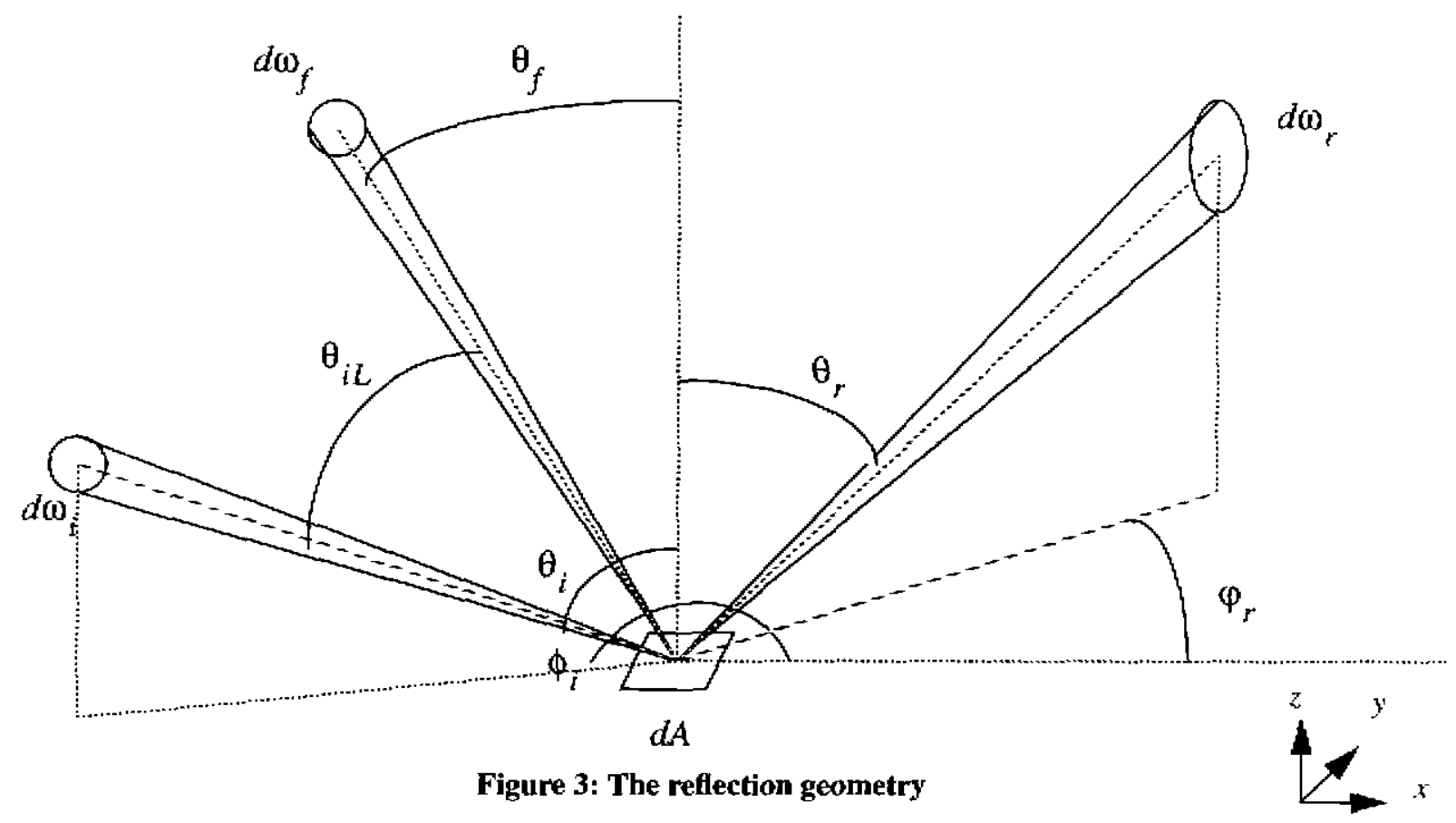

Assume an infinitesimal source of radiance $L_{i}$ subtending a solid angle d $\omega_{i}$ with direction of incidence, in spherical coordinates, $\left(\theta_{i}, \varphi_{i}\right)$. (The normal to the mean surface is in direction $(0,0)$.) The reflected radiance is $d L_{r}$, with direction $\left(\theta_{r}, \varphi_{r}\right)$. For a given incidence direction and reflectance direction only those facets whose normals bisect the angle between these directions will be able to reflect light from the source to the receiver. Let the direction of the normals to these facets be $\left(\theta_{f} \varphi_{f}\right)$. Let us denote the local angle of incidence, or the angle between the source direction and the direction of the normals to the privileged facets, as $\theta_{i L}$.

Now, in order to calculate the radiance of the patch, we must know the total area of the privileged facets, which will reflect light from the source to the receiver. We therefore define a function, $S\left(\theta_{f} \varphi_{f}\right)$, the facet area distribution function (FADF), which represents the total area of the privileged facets per unit solid angle subtended by the normals to these facets per unit area of the mean surface. Thus, the area of the facets present on an infinitesimal mean surface patch of area $d A$ with normals in the infinitesimal solid angle $d \omega_{f}$ is $S\left(\theta_{f} \varphi_{f}\right) d \omega_{f} d A$. The power incident on the privileged facets is then

$$
P_{i}=L_{i} d \omega_{i} \cos \theta_{i L} S\left(\theta_{f}, \varphi_{f}\right) d \omega_{f} d A
$$

This light will then reflect off the facets and into the receiver, and may be attenuated upon reflection. From the point 
of view of the receiver, the power received from the facets may be written as

$$
P_{r}=F G P_{i}=d L_{r} \cos \theta_{r} d \omega_{r} d A
$$

where $d L_{r}$ is the radiance of the patch as seen from the viewing direction, and $F$ is the Fresnel coefficient for the surface. The Fresnel coefficient will be a function of the local angle of incidence, $\theta_{i L}$. Some researchers[] also model the attenuation due to the shadowing of one facet by another, and quantify this with $G$, the geometrical attenuation factor. It is a function of the incident and exitant directions. Torrance and Sparrow provide a derivation of this factor. Substituting the expression for the incident power into the equation for the reflected power, we see that the radiance of the surface patch is

$$
d L_{r}=\frac{L_{j} d \omega_{i} \cos \theta_{i L} F G S\left(\theta_{f}, \varphi_{f}\right) d \omega_{f}}{\cos \theta_{r} d \omega_{r}}
$$

Most researchers use the simplifying relation $[8,12$

$$
d \omega_{f}=\frac{d \omega_{r}}{4 \cos \theta_{i L}}
$$

We, however, will most often use the relation

$$
d \omega_{i}=d \omega_{r}
$$

We have found that numerical methods of calculating the reflected radiance often perform better if we use this relation. Additionally, the equation for the reflected radiance has the same form for both the one-dimensional and twodimensional cases if we make this substitution.

For an extended source, the reflected radiance is then

$$
L_{r}=\iint \frac{L_{i} F G \cos \theta_{i L} S\left(\theta_{f}, \varphi_{f}\right) d \omega_{f}}{\cos \theta_{r}}
$$

where the integral is over the hemisphere for which $\theta_{f}$ is less than $\frac{\pi}{2}$.

There is also a normalization condition which any realistic FADF must satisfy. Consider one facet of the surface and let its area be $d F$. The area of the projection of this element onto the mean surface is

$$
d F \cos \theta_{f}
$$

If we were to add up all the projected areas of all facets on the surface, we would obtain $d A$, the area of the patch, i.e.

$$
d A=\int d F \cos \theta_{f}
$$

We also know that

$$
d F=S\left(\theta_{f} \varphi_{f}\right) d \omega_{f} d A
$$

so that

$$
d A=\int s\left(\theta_{\rho}, \varphi_{f}\right) \cos \theta_{f} d \omega_{f} d A
$$


and

$$
\int S\left(\theta_{f}, \varphi_{f}\right) \cos \theta_{f} d \omega_{f}=1
$$

which is the normalization condition.

\section{One-dimensional Gaussian heights}

\subsection{Theory}

The results so far pertain to any slope-based model, or FADF. In order to proceed further, we must now choose a specific model for our rough surface. Of particular interest is one which we call the "one-dimensional Gaussian height" distribution function. We believe this function approximately models surfaces which have been rolled or milled. It is also one of the more commonly used models. Consider a surface which varies in height along one direction, but which is constant in height along the perpendicular direction. The surface thus consists of a series of parallel grooves, Assume that the height values along the first axis are random and follow a Gaussian distribution. We wish to model this surface as a collection of facets. As shown by Beckmann and Spizzichino[1], such a surface is described by

$$
S\left(\theta_{f}\right)=\frac{e^{-\left(\frac{\tan \theta_{f}}{\beta}\right)^{2}}}{\beta \sqrt{\pi}\left(\cos \theta_{f}\right)^{3}}
$$

where $\beta$ is the roughness parameter, and $\theta_{f}$ is the angle between the normal to the surface patch and the facet normal. All the facet normals lie in a plane, and the standard deviation of the facet slopes is $\frac{\beta}{\sqrt{2}}$. Note that for this case, $d \omega_{t}$ is replaced by $d \theta_{f}$ in equation ( 6 ), since all the facets are coplanar and may be described by one variable. As noted by Beckmann and Spizzichino[1] and Nayar[8], the function may be approximated by a Gaussian in the limit of small $\beta$, and thus the model of Torrance and Sparrow, with its Gaussian distribution function, is a reasonable approximation to this function for the case of a fairly smooth surface. This facet area distribution function is more realistic than the Gaussian model, however, since it satisfies the normalization condition (equation (8)).

Now, let us determine the radiance pattern for the reflection of a step edge in a rough surface with the given facet area distribution function. We will perform our calculations in the coordinate system of the surface patch. Therefore, let the normal to the patch be along the z-axis, as shown in Figure 2, and let the grooves lie parallel to the $y$-axis (i.e. the height is constant for constant $x$ ). Let the source be a Lambertian half-plane of radiance $t$. Although this may seem to be a very restrictive assumption, because we are studying the reflected images in the regions local to edges, it is not excessively restrictive; if we wish to study the interaction of neighboring edges, we will have to drop the half-plane assumption. Let us first consider the easiest case, in which the view vector lies in the plane of the surface normals, i.e., the $x-z$ plane. Assume the edge of the source is parallel to the $y$-axis. We will remove these restrictions shortly. As we see in Figure 4, light is incident on the patch through a range of values of the angle $\theta_{i}$. Let $\theta_{i 0}$ denote the starting value and $\theta_{i 1}$ the ending value of this range. We also know the direction of the view vector, and hence $\theta_{r}$. for the patch. Because

$$
\theta_{f}=\frac{\theta_{j}+\theta_{r}}{2}
$$

we therefore know the range of values of $\theta_{f}$ for which light will reflect from the source into the camera. Let us denote 


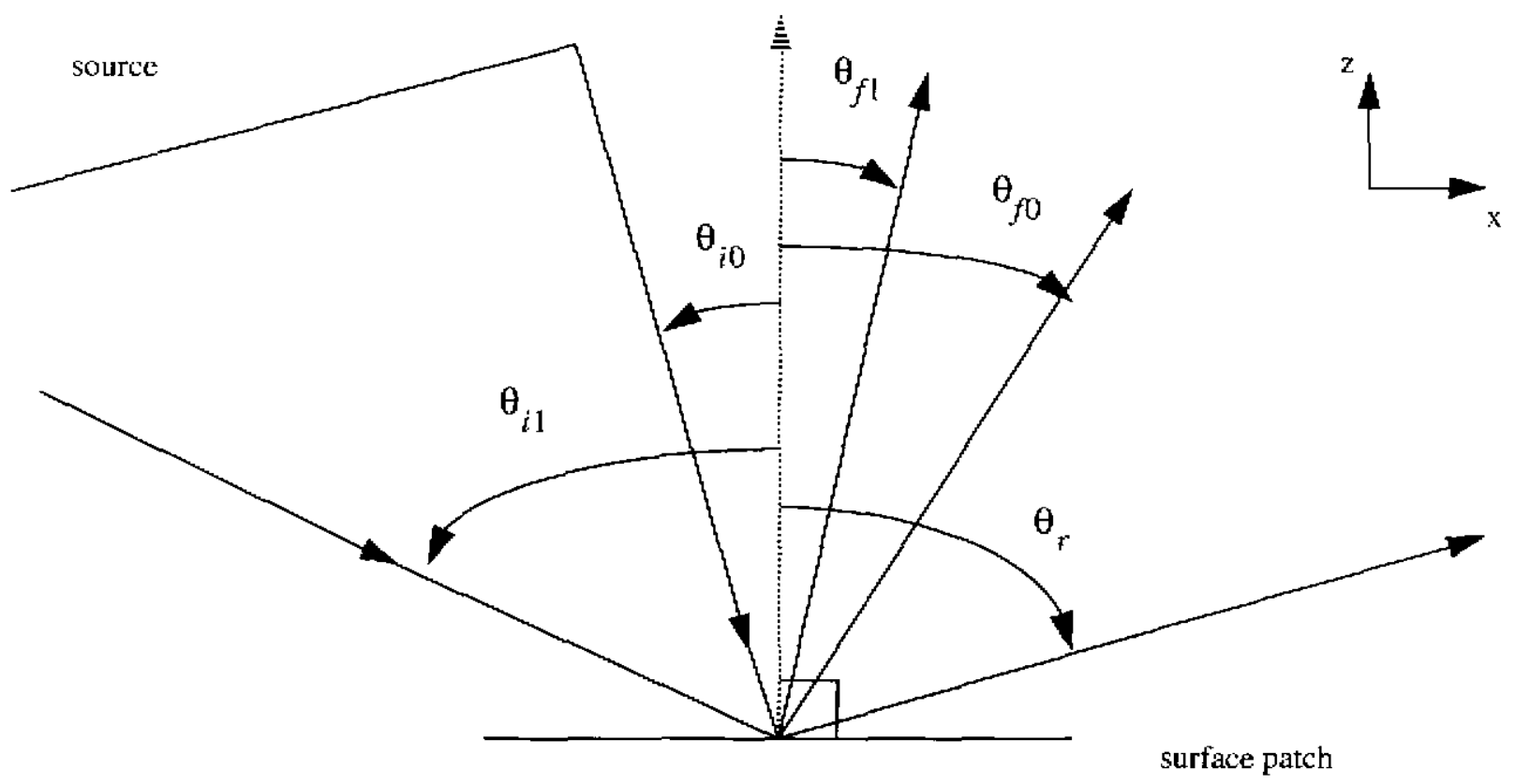

Figure 4: The one-dimensional reflection geometry

the starting and ending values of this range by $\theta_{f 0}$ and $\theta_{f 1}$, respectively. Now, let us return to equation (2), which gives the reflected radiance for a patch. We will make the simplifying assumptions that $F=1$ and $G=1$. The first assumption holds fairly well over a large range of angles for most metals. The second assumption holds when both $\theta_{i}$ and $\theta_{r}$ are less than approximately 45 degrees[8]. We expect some error due to this assumption, but not a substantial amount. With these assumptions, the reflected radiance will be given by

$$
L_{r}=\int_{\theta_{\beta 1}}^{\theta_{f 1} l \cos \theta_{i L} e^{-\left(\frac{\tan \theta_{f}}{\beta}\right)^{2}} d \theta_{f}} \frac{\sqrt{\pi}\left(\cos \theta_{f}\right)^{3} \cos \theta_{r}}{\beta(n)}
$$

Because the angle at which light is incident on a facet must equal the angle at which light exits the facet,

$$
\cos \theta_{i L}=\cos \left(\theta_{i}-\theta_{f}\right)=\cos \left(\theta_{f}-\theta_{r}\right)=\cos \theta_{f} \cos \theta_{r}+\sin \theta_{j} \sin \theta_{r}
$$

and thus

$$
L_{r}=\int_{\theta_{f 0}}^{\theta_{f 1}}\left(\frac{l e^{-\left(\frac{\tan \theta_{f}}{\beta}\right)^{2}} d \theta_{f}}{\beta \sqrt{\pi}\left(\cos \theta_{f}\right)^{2}}+\frac{l e^{-\left(\frac{\tan \theta_{f}}{\beta}\right)^{2}} \tan \theta_{f} \tan \theta_{r} d \theta_{f}}{\beta \sqrt{\pi}\left(\cos \theta_{f}\right)^{2}}\right)
$$

and 


$$
L_{r}=\frac{l}{2}\left(\operatorname{erf}\left(\frac{\tan \theta_{f 1}}{\beta}\right)-\operatorname{erf}\left(\frac{\tan \theta_{f 0}}{\beta}\right)-\frac{\beta}{\sqrt{\pi}} \tan \theta_{r}\left(e^{-\left(\frac{\tan \theta_{f 1}}{\beta}\right)^{2}}-e^{-\left(\frac{\left.\tan \theta_{j 0}\right)^{2}}{\beta}\right)}\right)\right)
$$

The radiance of the reflected image will change from point to point, since the values of $\theta_{f 0}, \theta_{f 1}$, and $\theta_{s}$ will change from point to point on the surface.

Now let us remove the assumptions that the edge of the source is parallel to the grooves of the rough reflecting surface, and that the view vector lies in the $\mathrm{x}-\mathrm{z}$ plane. We note that the rough surface is still parametrize by a single variable, $\theta_{f}$, so that we may still calculate the reflected radiance by means of equation (11). Note, however, that for this case, $\cos \theta_{i L}$ is given by

$$
\cos \theta_{i L}=\cos \theta_{f} \cos \theta_{r}+\sin \theta_{f} \sin \theta_{r} \cos \left(\varphi_{f}-\varphi_{r}\right)
$$

Because the surface is "one-dimensionally" rough, however, $\varphi_{f}=0$, and thus

$$
\cos \theta_{i L}=\cos \theta_{f} \cos \theta_{r}+\sin \theta_{f} \sin \theta_{r} \cos \varphi_{r}
$$

so that

$$
L_{r}=\frac{l}{2}\left(\operatorname{erf}\left(\frac{\tan \theta_{f 1}}{\beta}\right)-\operatorname{erf}\left(\frac{\tan \theta_{f 0}}{\beta}\right)-\frac{\beta}{\sqrt{\pi}} \tan \theta_{r} \cos \varphi_{r}\left(e^{-\left(\frac{\tan \theta_{f 1}}{\beta}\right)^{2}}-e^{-\left(\frac{\tan \theta_{j 0}}{\beta}\right)^{2}}\right)\right)
$$

This description of the reflection of light from rough surfaces is not complete, however. As shown by Beckmann and Spizzichino, there is a purely specular component of reflection which can only be explained by considering the wave nature of the light. Nayar[8] calls this component the "specular spike component of reflection", and that described by equation (15) as the "specular lobe component". Apart from a multiplicative constant, the spike component of reflection is the same as the reflection that would occur if the surface were completely smooth. Thus, we model the light reflected from the rough surface as

$$
L_{r}=A\left(\frac{l}{0}\right)+B\left(\frac{l}{2}\left(\operatorname{erf}\left(\frac{\tan \theta_{f 1}}{\beta}\right)-\operatorname{erf}\left(\frac{\tan \theta_{f 0}}{\beta}\right)-\frac{\beta}{\sqrt{\pi}} \tan \theta_{r} \cos \varphi_{r}\left(e^{-\left(\frac{\tan \theta_{f 1}}{\beta}\right)^{2}}-e^{-\left(\frac{\tan \theta_{f 0}}{\beta}\right)^{2}}\right)\right)\right)
$$

where $A$ and $B$ describe the relative intensities of the two components, and $\left(\begin{array}{l}l \\ 0\end{array}\right)$ means that the intensity is non-zero only if the light may reflect specularly from the source to the receiver.

\subsection{Experiment}

We tested the correctness of the theory by calculating the roughness parameter for several surfaces. The samples were circular stainless steel disks, three inches in diameter, which had been milled to produced a one-dimensionally rough surface on one face of each disk. The values of the arithmetic average roughness, $R_{A}[11]$, as stated by the manufacturer were 1.5, 3.0, 4.5, 7.5, and 9.5 microinches. The camera was a 12-bit Photometrics Star 1 camera. A large piece of white paper was used as the step edge source. Because the source had a much greater area than the steel plates, it was an appropriate choice to approximate a step edge. Figure 5 shows the experimental configuration. The distance from the disks to the camera was approximately $120 \mathrm{~cm}$, and the distance from the plane of the samples to the plane of the source was $9.57 \mathrm{~cm}$. The viewing angle, $\theta_{r}$, was 7.29 degrees. 

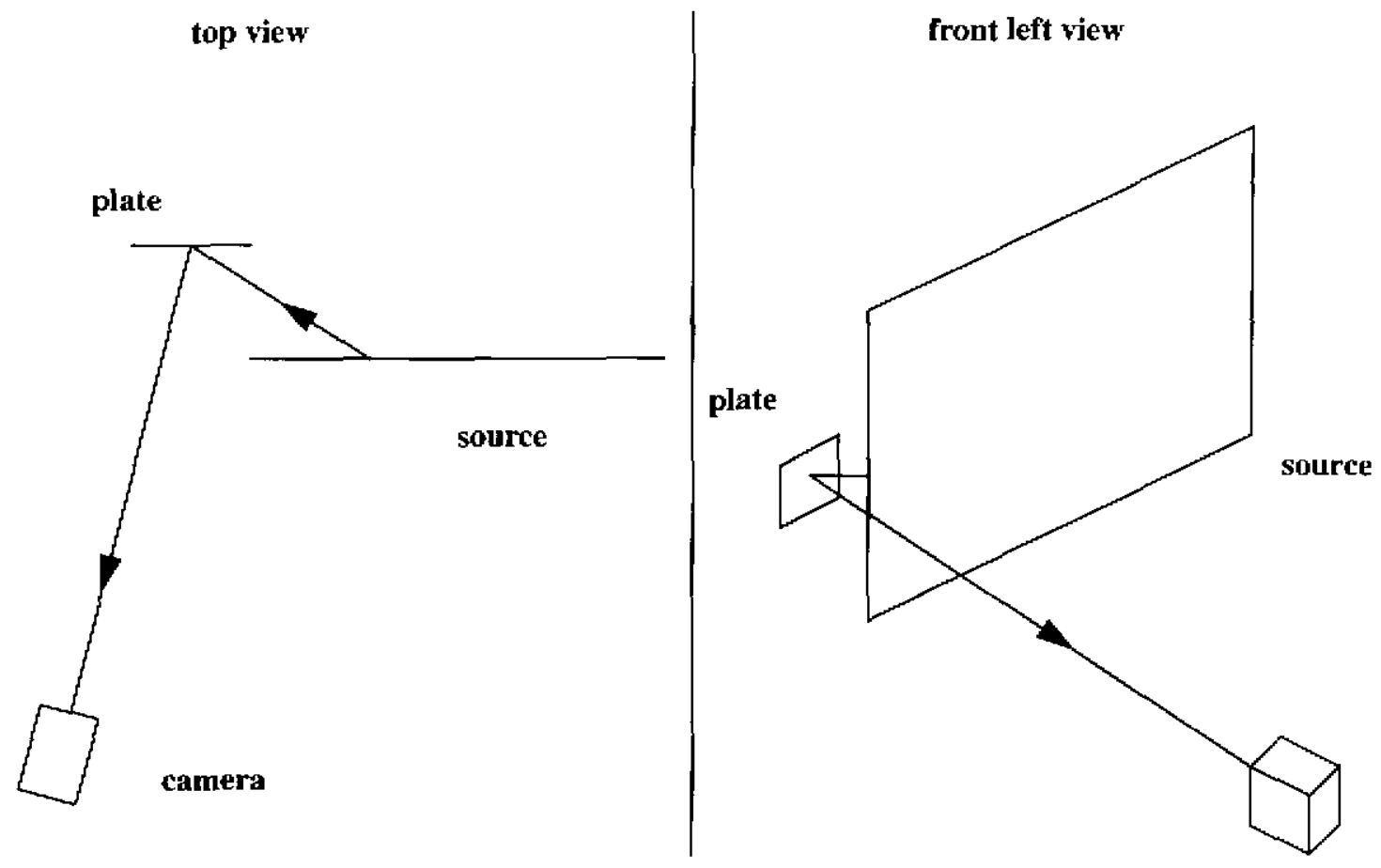

Figure 5: Schematic of the experimental configuration

In order to apply the results of the theory, the intensity of the reflected step edge should be studied along the direction perpendicular to the reflected edge. For a generic configuration of source, reflective surface, and camera, it would therefore be necessary to find the reflected edge and determine its direction. This is not difficult in general, and would be the first step in a roughness estimation vision system. We felt that the extra calculation involved in edge detection would add nothing to the test of the theory, and thus the reflected edge was aligned vertically in the image. Similarly, the grooves of the rough surface lay along this direction. This was done merely to simplify the calculations, and in no way represents a loss of generality in the test of the theory. Note that since we assume an orthographic projection, $\varphi$. is a constant for all points on the reflective surface. For this configuration, $\varphi_{r} \approx 0$, and equation (15) reduces to equation (13). We may derive all the necessary terms in this equation from Figure 6 . First, let us find the values of $\theta_{f 0}$ and $\theta_{f 1}$ for every point on the steel plate. Let $x_{s r c}$ be a point on the source, and $x_{o b j}$ be a point on the object. We see that

$$
\theta_{i}=\operatorname{atan}\left(\frac{x_{s r c}-x_{o b j}}{D}\right)
$$

where $D$ is the distance between the source plane and the object plane. Also,

$$
\theta_{f}=\frac{\theta_{i}+\theta_{r}}{2}=\frac{\operatorname{atan}\left(\frac{x_{s r c}-x_{o b j}}{D}\right)+\theta_{r}}{2}
$$

We therefore know the initial and final values of $\theta_{f}$ for all points on the object, and we may therefore use equation (13).

The images used as data appear in Figure 7 to Figure 9 in order of increasing roughness. The appearance of the reflected step edges is as one would expect from intuition. 


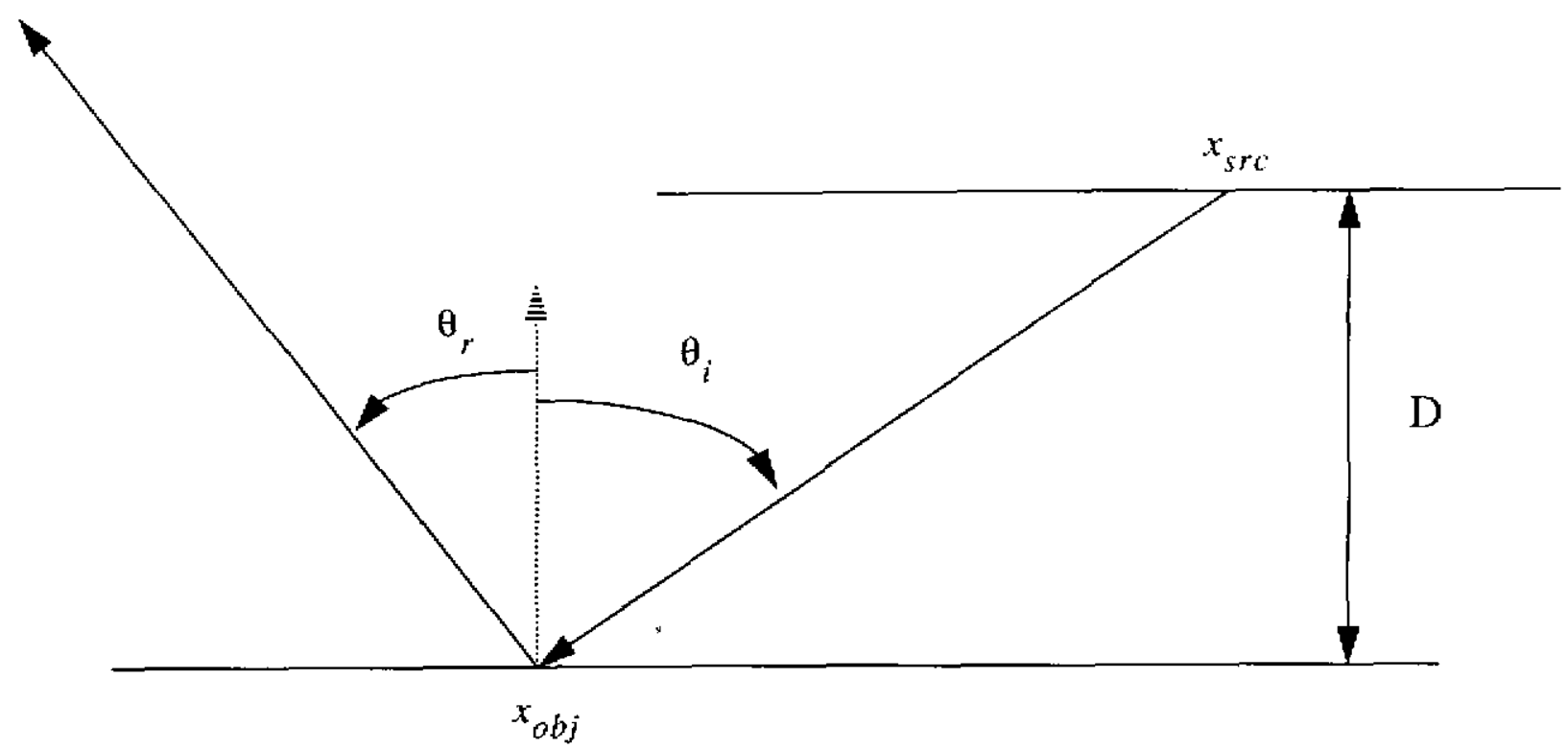

Figure 6: The reflection geometry for the experimental configuration
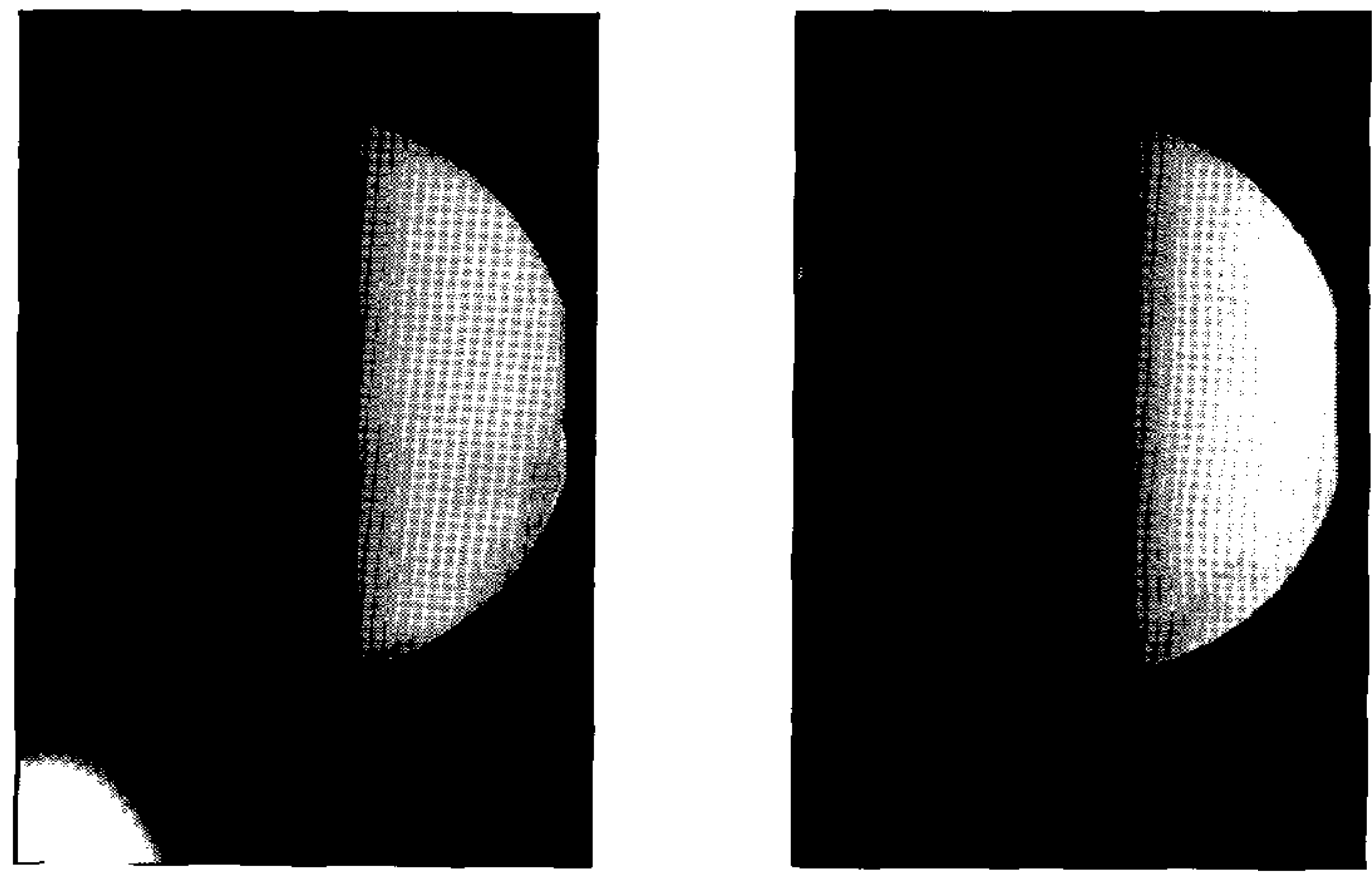

Figure 7: The reflected image of the step edge in the 1.5 microinches $R_{A}$ sample and the 3.0 microinches $R_{A}$ sample

Now, in order to fit the model to the data, we must account for several effects which change the appearance of the reflected image, but which are not included in equation (13). First, note that the dimmest parts of the image do not have a zero intensity, but rather there appears to be a constant offset due to ambient light and perhaps camera effects. We model this with the constant, $C$. Next, note that the value given for $\theta_{r}$ is the result of a measurement and is there- 

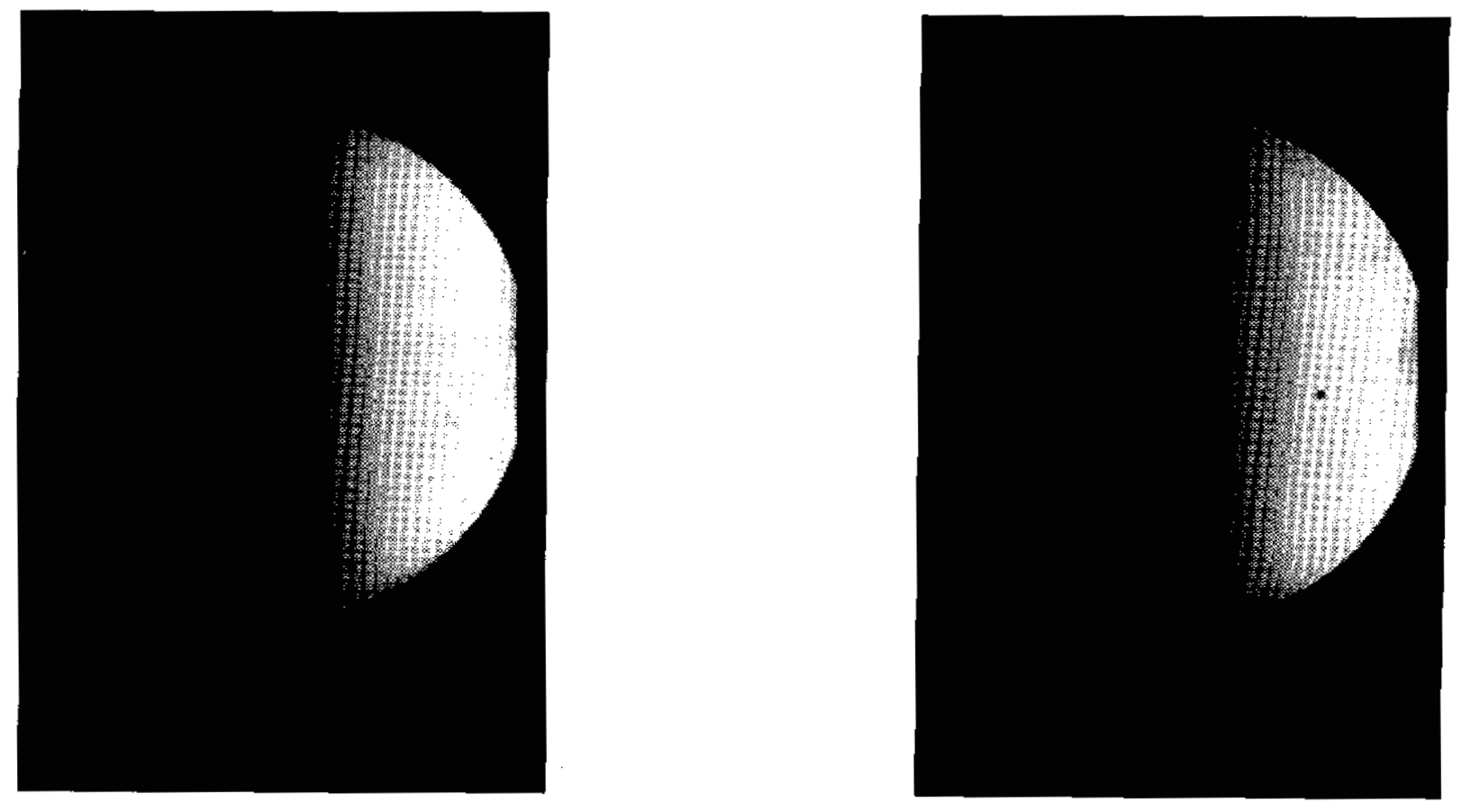

Figure 8: The reflected image of the step edge in the 4.5 microinches $R_{A}$ sample and the 7.5 microinches $R_{A}$ sample

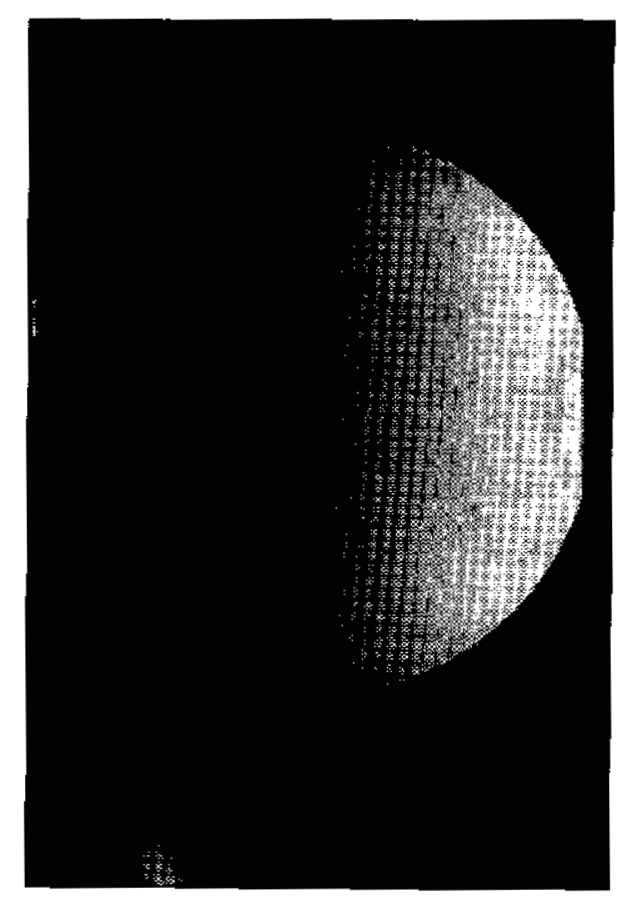

Figure 9: The reflected image of the step edge in the 9.5 microinches $R_{A}$ sample fore not exact; the same is true for $\theta_{i}$. Apart from the term $\tan \theta_{r}$, which is a small number for this experimental con- 
figuration and which is multiplied by other small numbers, $\theta_{r}$ and $\theta_{i}$ only appear as part of their sum. Therefore, we may model the errors in both $\theta_{r}$ and $\theta_{i}$ by replacing $\theta_{r}$ with $\theta_{r}+\Delta \theta$. The final model for the reflected edge is then

$$
L_{r}\left(x_{o b j}\right)=A\left(\frac{l}{0}\right)+B \frac{l}{2}\left(\operatorname{erf}\left(\frac{\tan \theta_{f 1}}{\beta}\right)-\operatorname{erf}\left(\frac{\tan \theta_{f 0}}{\beta}\right)-\frac{\beta}{\sqrt{\pi}} \tan \left(\theta_{r}+\Delta \theta\right)\left(e^{-\left(\frac{\tan \theta_{f 1}}{\beta}\right)^{2}}-e^{-\left(\frac{\tan \theta_{f 1}}{\beta}\right)^{2}}\right)\right)+C
$$

where the first component was $A l$ for $\theta_{i 0} \leq \theta_{r}<\theta_{i 1}$, and 0 otherwise. This term models the specular spike component of reflection and is simply the reflected image which would have occurred had the surface been mirror-like. The constant $B$ gives the strength of the specular lobe component, and the other terms are as described above. We allow the constant $l$ to equal one, since the terms $A$ and $B$ can model any changes in the value of $l$. Our goal is to find the best fit values of $A, B, C, \beta$, and $\Delta \theta$ for the data.

In order to mitigate the effects of noise, the scanlines of each image were averaged to yield a representative reflected 
edge, which was then the data for all calculations. Figure 10 to Figure 14 show these average scanlines. The values of

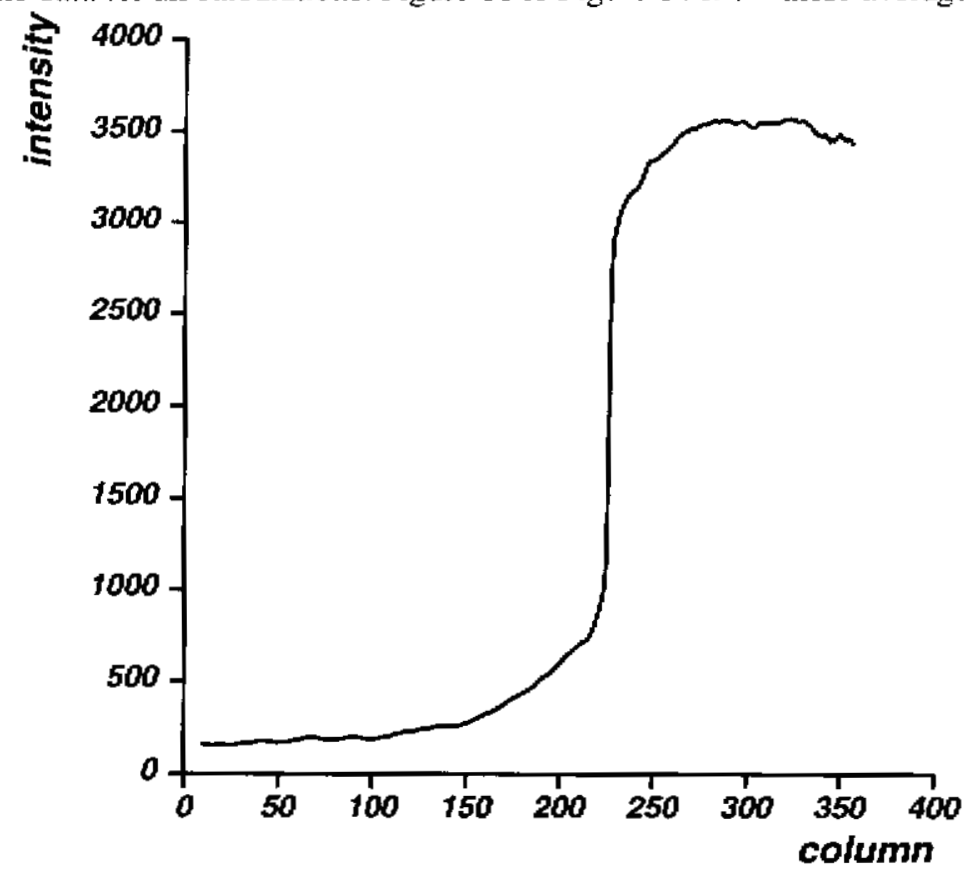

\section{5 microinches roughness}

Figure 10: The average of the scanlines

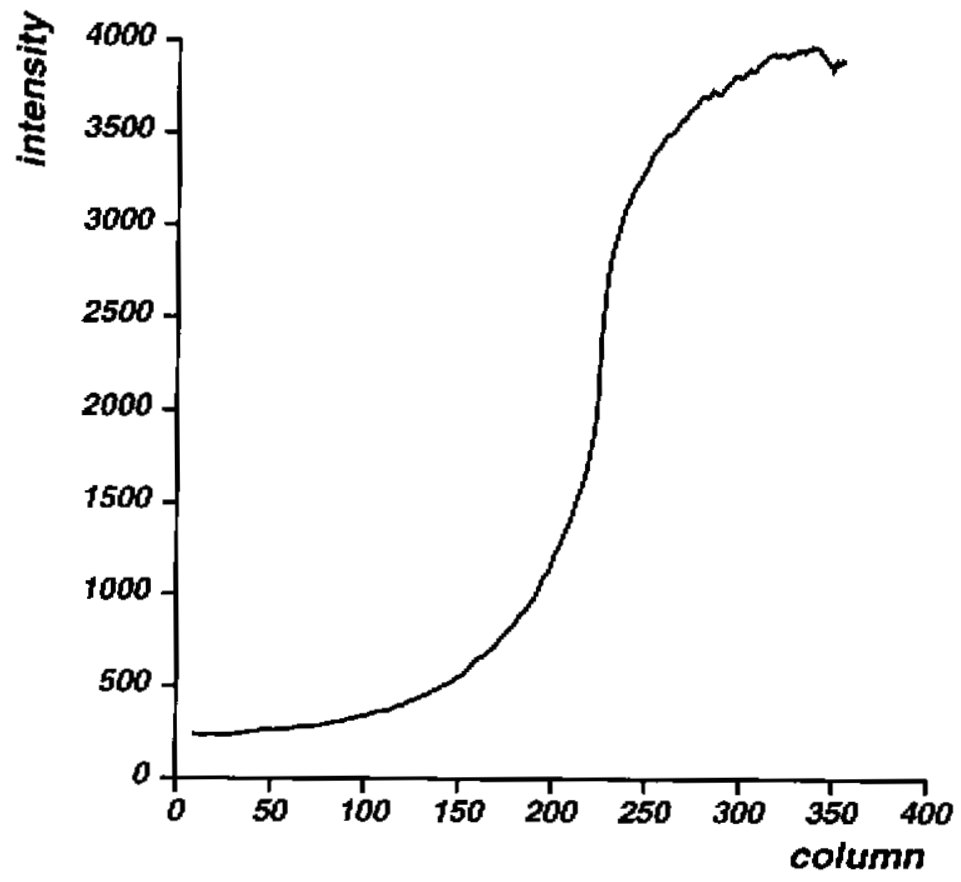

3.0 microinches roughness

Figure 11: The average of the scanlines

the parameters were found as follows. First, the minimum value of the reflected edge was taken as the initial guess for $C$. Next, a step edge with minimum value $C$ and maximum value equal to the maximum value of the reflected edge 


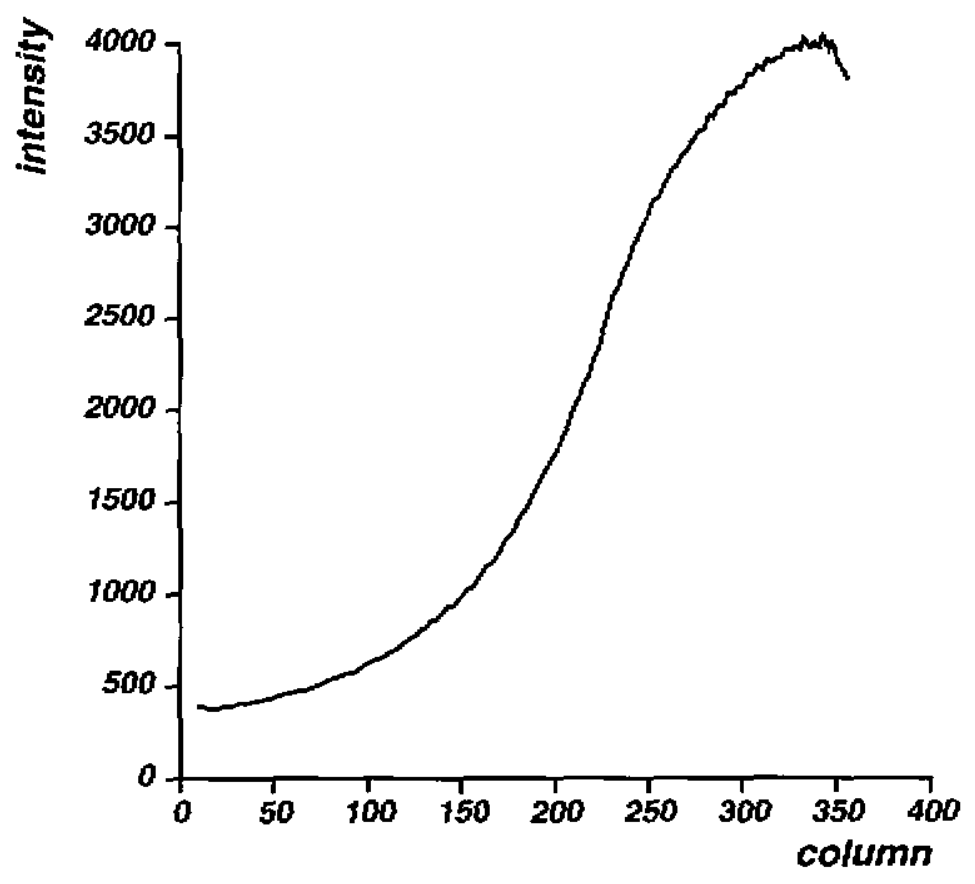

\section{5 microinches roughness}

Figure 12: The average of the scanlines

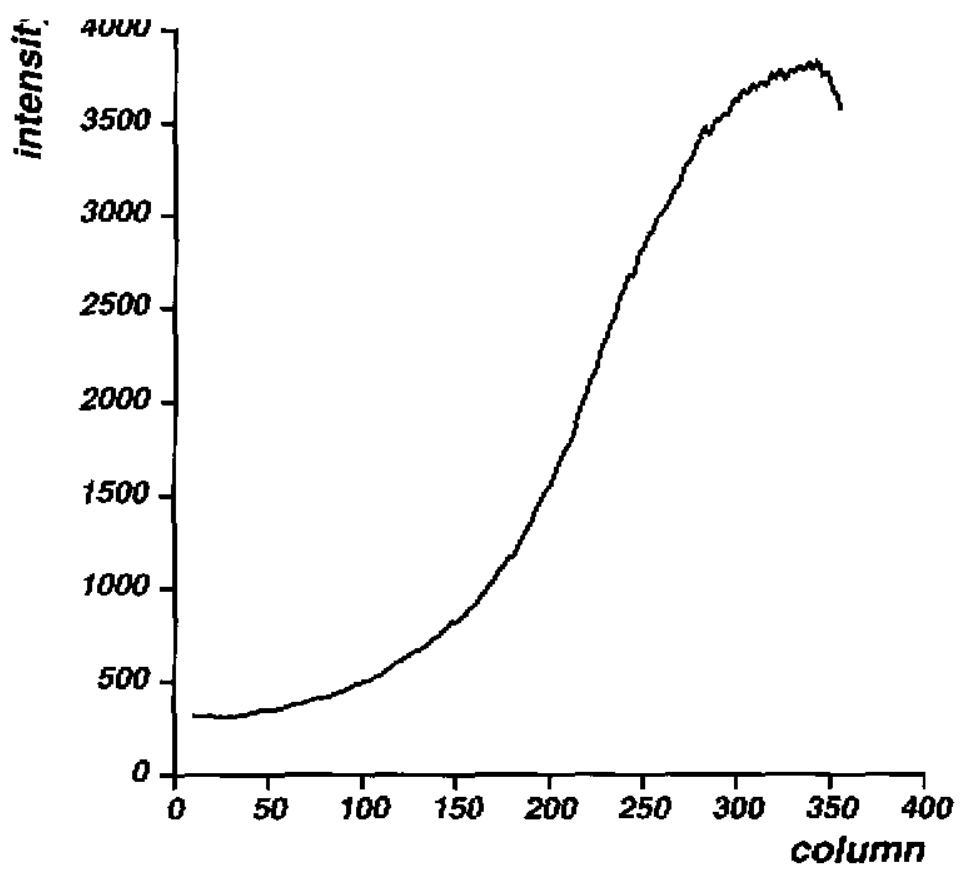

\section{5 microinches roughness}

Figure 13: The average of the scanlines

was formed. The sum of squared differences between this edge and the reflected edge curve was found for different values of $\Delta \theta$. The minimum of the sum of squared differences with respect to $\Delta \theta$ was found by bracketing and bisection, and the value of $\Delta \theta$ which gave this minimum was taken as the best fit value of $\Delta \theta$. Next, the pixel at 


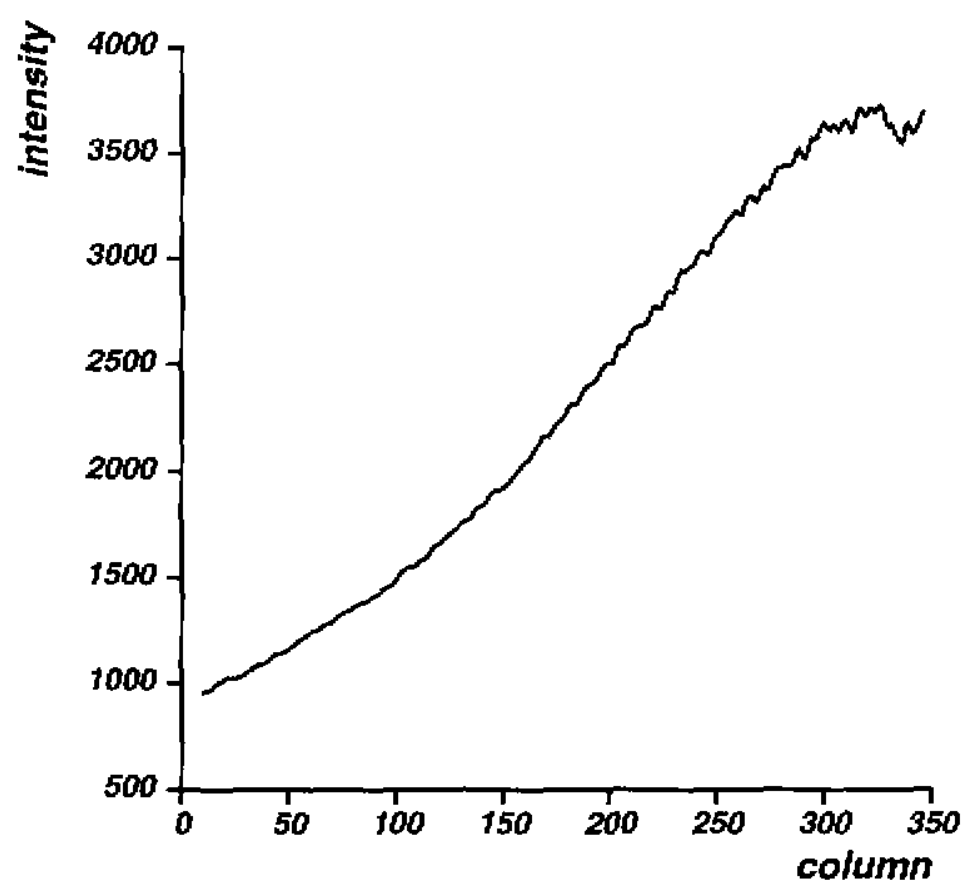

\section{5 microinches roughness}

Figure 14: The average of the scanlines

which the discontinuity in the step edge occurred was found. The intensities a few (usually three) pixels on either side of the central pixel were recorded, and their difference was taken as the initial value of $A$. The first guess for $B$ was then the maximum value of the reflected edge, minus $C$, minus $A$.

Once the initial values for $A, B$, and $C$ were determined, the sum of squared differences between the predicted and measured curves could be found for a given $\beta$, and the best fit value of $\beta$ could be found by gradient descent. When this reached a minimum, singular value decomposition was used to find the best fit values of $A, B$, and $C$ for this value of $\beta$. The algorithm alternated between fitting for $\beta$ and fitting for $A, B$, and $C$ until convergence was reached. The best fit values for each sample are shown in Table 1; as we noted earlier, we may calculate the standard deviation for the facet area distribution function from $\beta$, and the last column of Table 1 shows these values in units of degrees. The corresponding curves and the data are shown in Figure 15 through Figure 19. The solid lines represent the fitted 
curve, and the dashed lines show the average scanline plus and minus the error bars.

Best fit values of the parameters

\begin{tabular}{lcccccc} 
sample & A & B & C & $\Delta \theta$ & $\beta$ & std. dev \\
\hline 1.5 microinches & 2300 & 1290 & 171 & -0.0272 & 0.0770 & 3.12 \\
\hline 3.0 microinches & 1220 & 2670 & 251 & -0.0312 & 0.0850 & 3.44 \\
\hline 4.5 microinches & 298 & 3510 & 373 & -0.0440 & 0.102 & 4.13 \\
\hline 7.5 microinches & 311 & 3360 & 299 & -0.0368 & 0.0990 & 4.01 \\
\hline 9.5 microinches & 109 & 3750 & 318 & -0.104 & 0.210 & 8.51 \\
\hline
\end{tabular}

Table 1

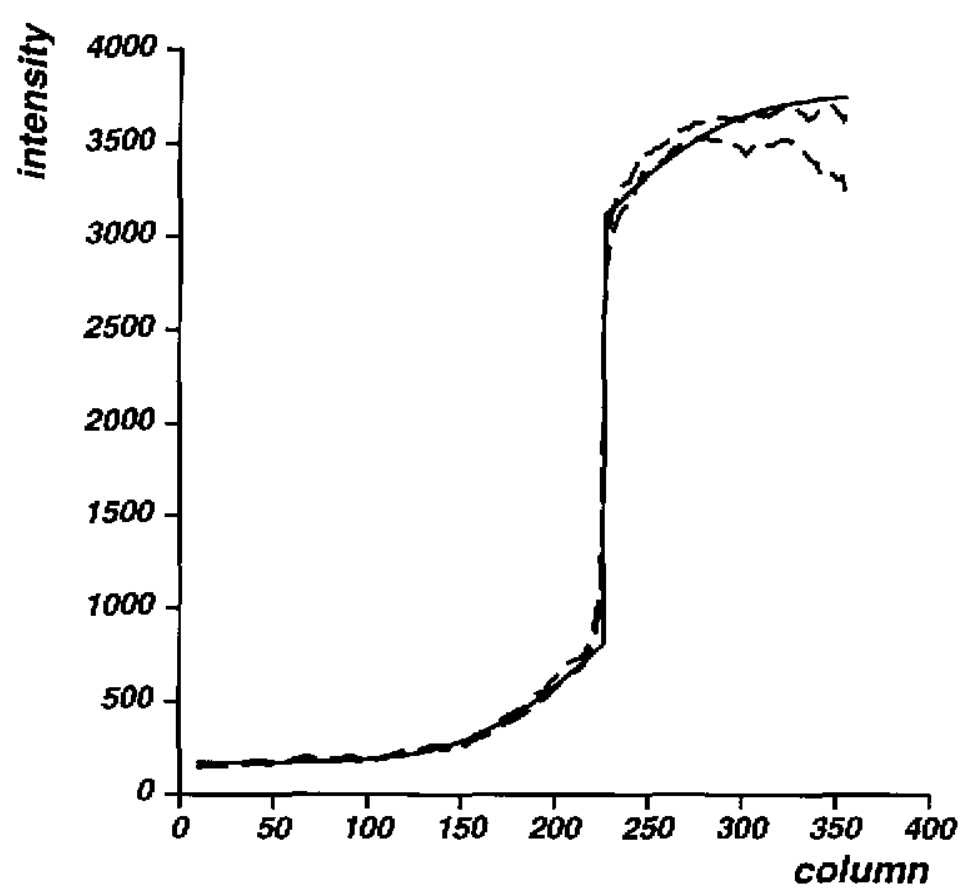

1.5 microinches roughness

Figure 15: The best fit scanline and error bars

We see that the fitting process orders the samples by roughness, except for the 7.5 microinches roughness sample. We will discuss possible reasons for this error shortly. The values found for $\beta$ are reasonable, and are similar to those 


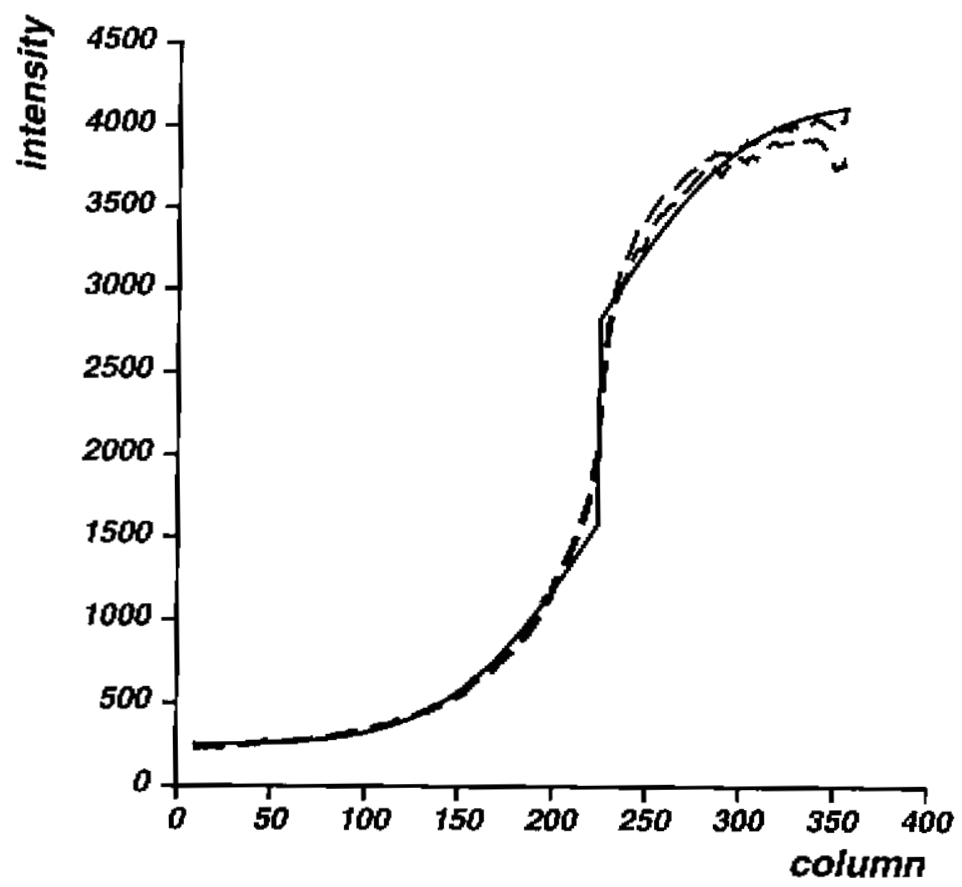

\section{0 microinches roughness}

Figure 16: The best fit scanline and error bars

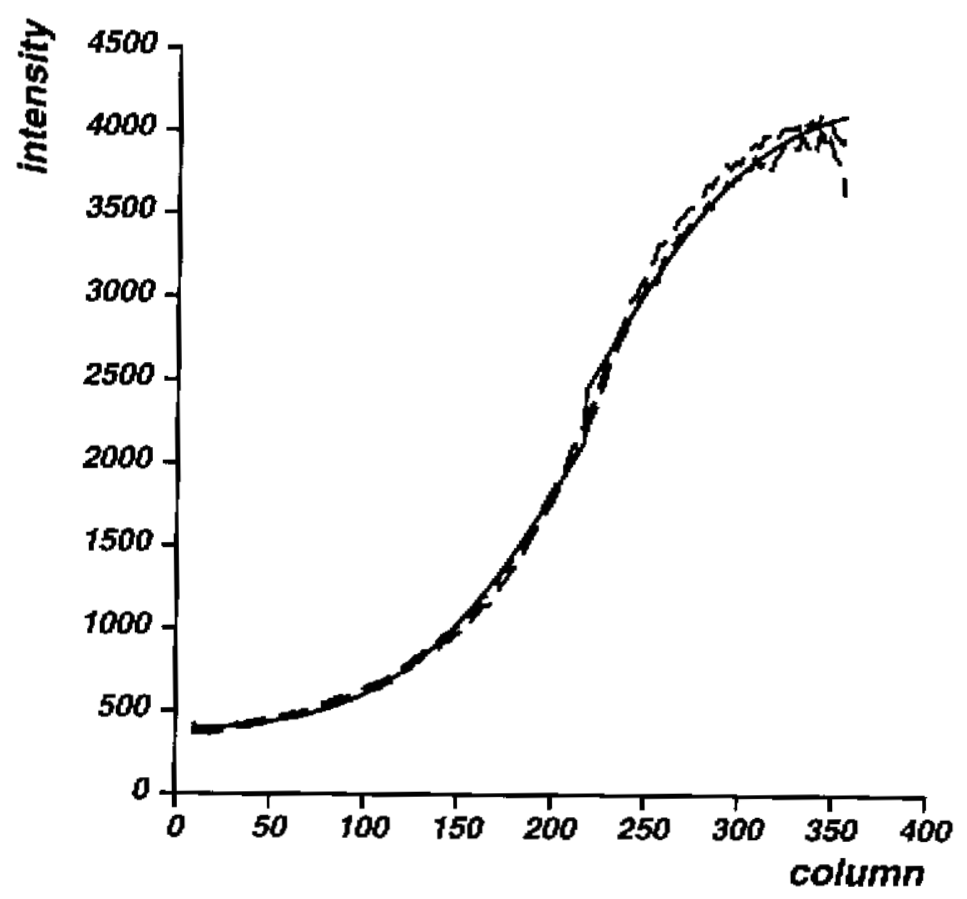

\section{5 microinches roughness}

Figure 17: The best fit scanline and error bars

found by other computer vision researchers [9]. Although the fitting process gives results consistent with our intuition, we must check the goodness of the fit, and compare the results with roughness measurements made by other methods. 


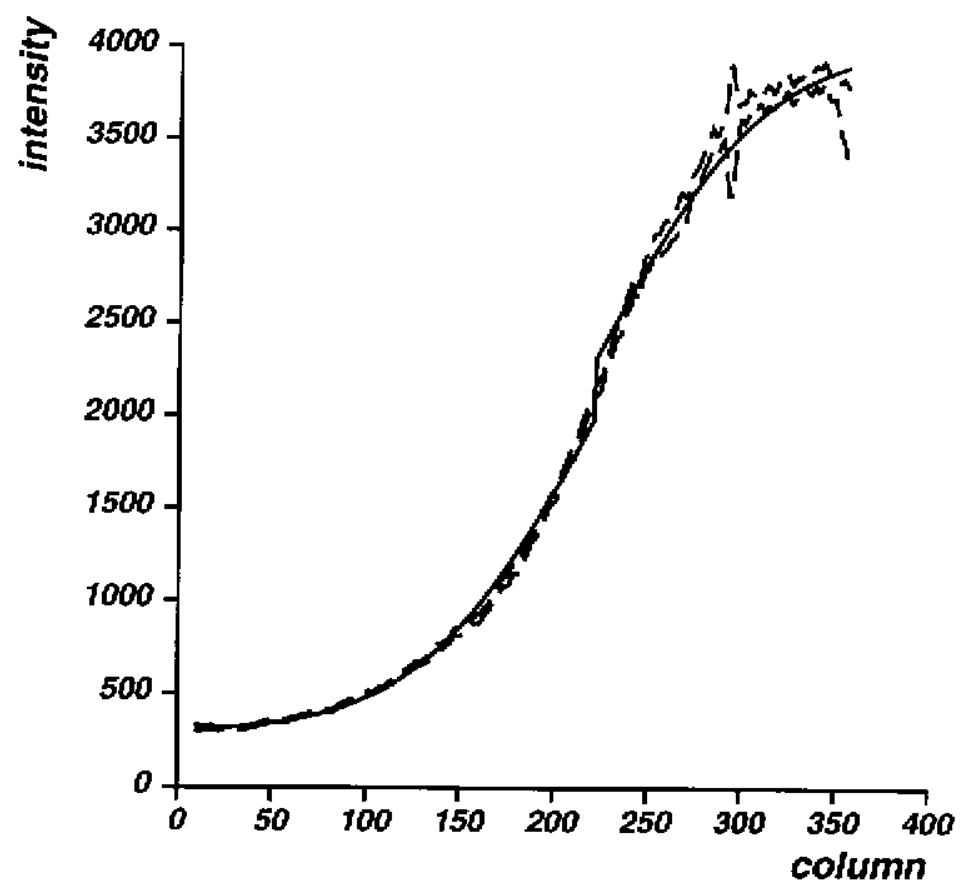

7.5 microinches roughness

Figure 18: The best fit scanline and error bars

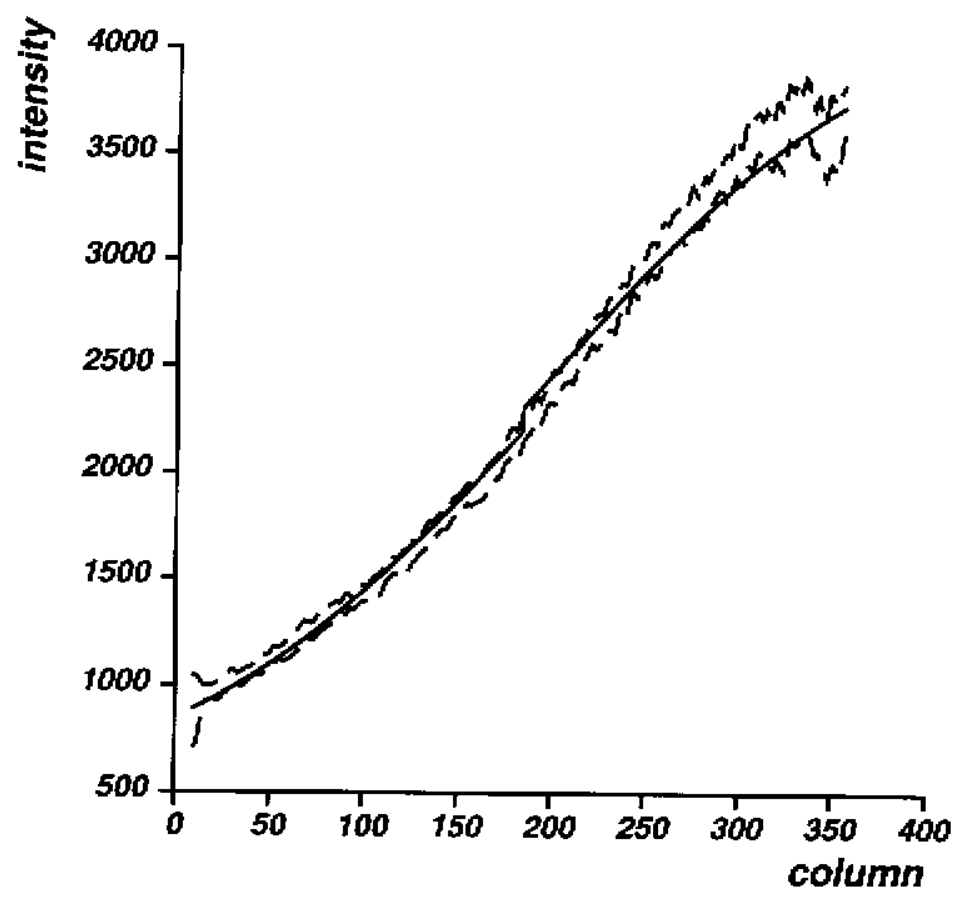

\section{5 microinches roughness}

Figure 19: The best fit scanline and error bars 
The best fit curves were compared to the data, and the statistics quantifying the goodness of fit are shown in Table 2 .

\section{Chi squared value for the fit}

\begin{tabular}{lcc} 
sample & $\chi^{2}$ & $\Gamma$ \\
\hline 1.5 microinches & 174191 & 0.000000 \\
\hline 3.0 microinches & 398126 & 0.000000 \\
\hline 4.5 microinches & 181983 & 0.000000 \\
\hline 7.5 microinches & 222623 & 0.000000 \\
\hline 9.5 microinches & 87473 & 0.000000 \\
\hline
\end{tabular}

Table 2

Although the curves appear reasonable, but not exact, the fits are not acceptable. Note that the error bars are probably too large, since we averaged over many scanlines, and thus included variations in the source intensity in our calculations of the error. Thus, the fits are certainly unacceptable for the more realistic case in which the errors are smaller. There are several possible reasons for the poor quality of the fits. First, the model of the reflected light, equation (13), is technically incorrect. Equation (13) should only be used for very rough surfaces (those with $\beta$ » 1) [1]. In this case, there should be no specular spike component, and thus, for all our samples which showed an appreciable spike component, equation (13) is not appropriate. Notice that the value of $\chi^{2}$ is lowest for the 9.5 microinches sample, presumably because the model is more appropriate for the rougher surface. Another possible source of error is the chance that the surface has a non-Gaussian distribution of heights. We will discuss this possibility after we compare our results with roughness measurements made by other methods.

Although our method produces roughness estimates comparable to those of the methods of other computer vision researchers, we wish to determine if the calculated roughness corresponds to the roughness for the surface as measured by conventional means. Before we describe our experiments, however, we mention a caveat. As noted by Bennett and Mattson[2], different values of surface roughness will be obtained for the same surface with different instruments. As a result, stylus profilometers generally measure higher values for the roughness of a surface than do optical profilometers. Keeping this in mind, we performed the following measurements. A Tencor Alpha Step 200 stylus profilometer was used to measure the surface profiles of the samples. For each scan, the profilometer measured the surface height at 2000 points over a 400 micrometer distance, and ten scans were made for each sample. The approximation of the surface by facets is a difficult problem; however, for a surface with a Gaussian distribution of heights, we know that the facet area distribution function for such a surface is[1]

$$
S\left(\theta_{f}\right)=\frac{T}{2 \sigma \sqrt{\pi}\left(\cos \theta_{f}\right)^{3}} e^{-\frac{T^{2}\left(\tan \theta_{f}\right)^{2}}{4 \sigma^{2}}}
$$


where $\sigma$ is the rms height and $T$ the correlation length of the surface. Therefore, $\beta=\frac{2 \sigma}{T}$. The calculated rms roughness values appear in Table 3. The values in microinches are given for comparison with the stated roughness

\title{
Measured rms roughness value
}

sample

\author{
rms roughness \\ micrometers microinches
}

$\begin{array}{lll}1.5 \text { microinches } & 0.0366 \pm 0.0002 & 1.44\end{array}$

$\begin{array}{lll}3.0 \text { microinches } & 0.0690 \pm 0.0003 & 2.72\end{array}$
4.5 microinches
$0.136 \pm 0.001$
5.34

7.5 microinches

$0.241 \pm 0.001$

9.48

\section{Table 3}

values. Remember that the roughness values stated by the manufacturer were the arithmetic roughness, rather than the root mean square roughness, and therefore need be the same as the values we calculate. Notice the unusually high value for the 7.5 microinches roughness sample. Note that, except for the fourth sample, the rms values place the samples in the same order as did the edge fitting method, and simple intuition. Next, the correlation lengths of the surfaces were found by taking the autocorrelation of each profile, and then fitting a function of the form

$$
e^{-\left(\frac{x}{T}\right)^{2}}
$$

in the least squares sense to each of the autocorrelation functions. The correlation lengths for the surfaces appear in Table 4. Again, notice the large value for the 7.5 microinches roughness sample. Graphs of the scans from the profilometer confirm that this sample has a much lower fundamental frequency for its surface than do the other samples. These two high values could explain why the facet area distribution function for this sample is almost the same as that for the 4.5 microinches sample, rather than showing a broader distribution. The standard deviations of the slope dis- 


\section{Calculated correlation length}

sample correlation length (micrometers)

1.5 microinches

$5.8 \pm 2.7$

3.0 microinches

$8.6 \pm 3.8$

4.5 microinches

$5.1 \pm 1.2$

7.5 microinches

$21.2 \pm 2.4$

9.5 microinches

$4.2 \pm 0.8$

Table 4

tributions are shown in Table 5. As can easily be seen, the values found by this method do not correspond at all to

\section{Standard deviation of the slope distribution}

sample

standard deviation (degrees)

1.5 microinches

$0.509 \pm 0.237$

3.0 microinches

$0.652 \pm 0.230$

4.5 microinches

$2.14 \pm 0.61$

7.5 microinches

$0.920 \pm 0.149$

9.5 microinches

$4.86 \pm 1.22$

\section{Table 5}

those found by the optical method and listed in Table 3. This discrepancy could be due to the poor quality of the estimates of the correlation length, or to the fact that two different methods of measurement were used for these calculations. Also, it is possible that the surfaces do not exhibit a Gaussian distribution of heights, and that the facet model of reflection is not applicable to these samples. We study this possibility next. 
Figure 20 to Figure 24 show the height histograms for the five samples, as determined by the profilometer data.

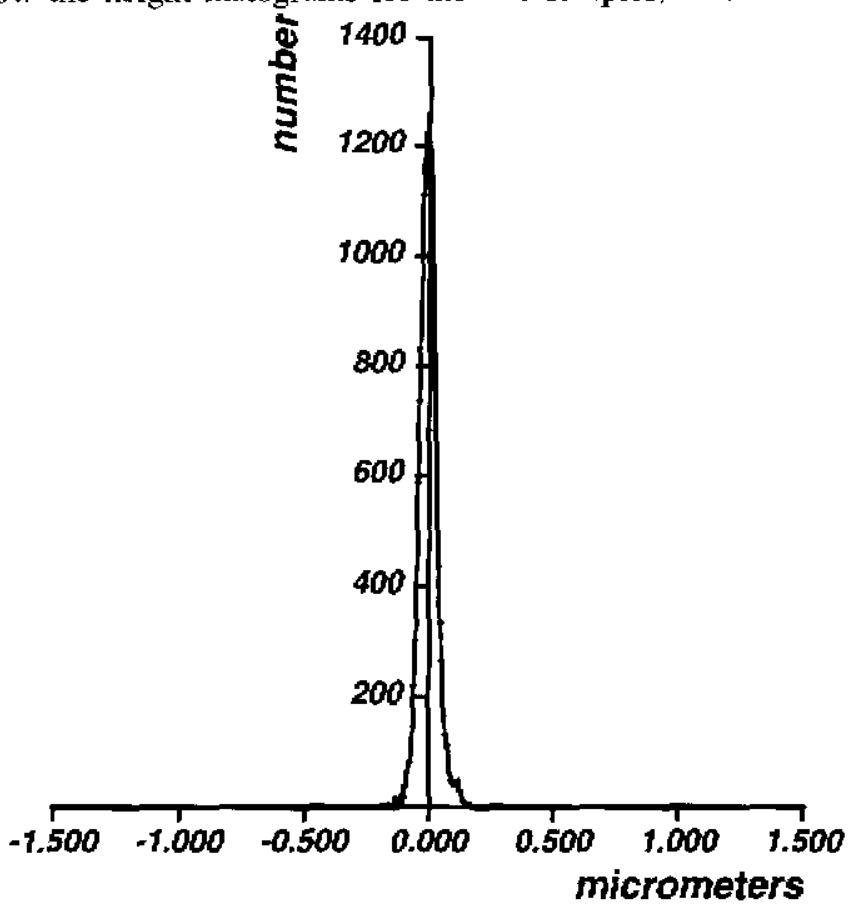

1.5 microinches sample

Figure 20: Number of occurrences of each height value

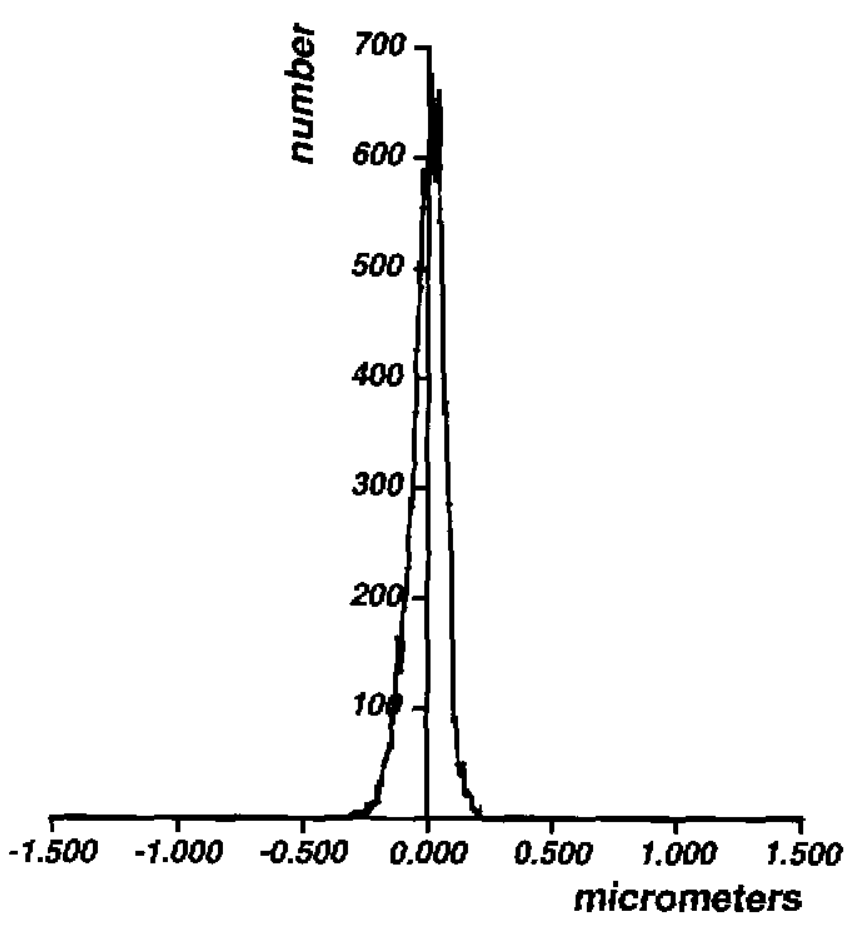

3.0 microinches sample

Figure 21: Number of occurrences of each height value

Except for the 7.5 microinches sample, the distributions appear reasonably Gaussian. We must, however, test to see if this guess is correct. The number of measured height values that fall within a certain range is given by a binomial dis- 


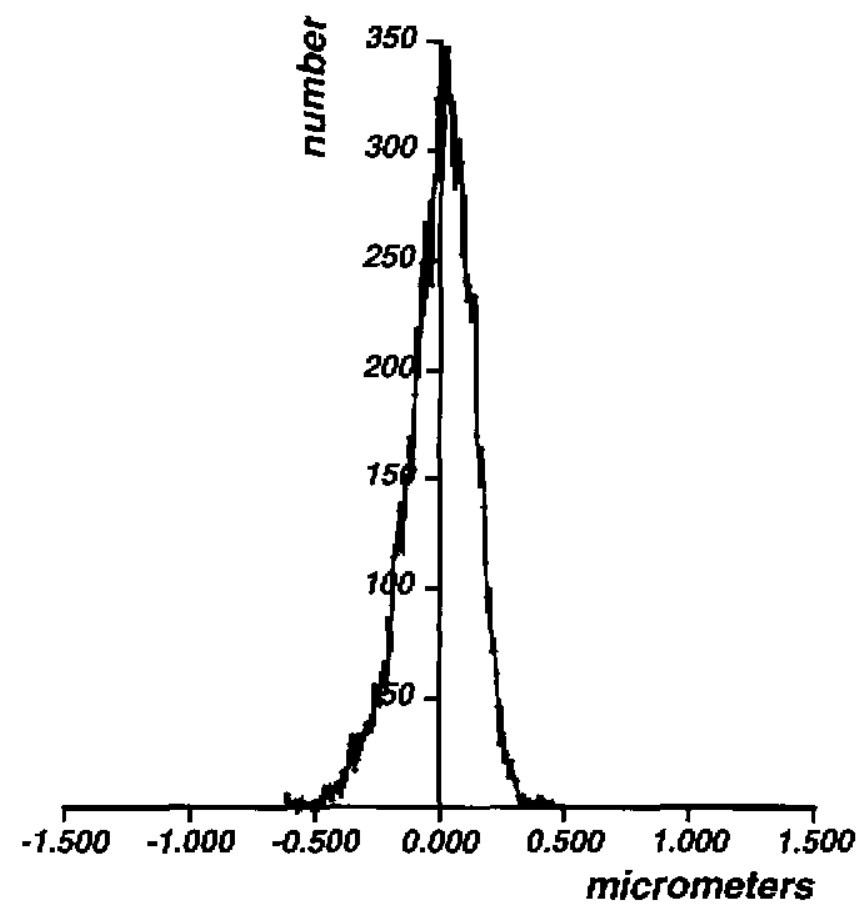

4.5 microinches sample

Figure 22: Number of occurrences of each height value

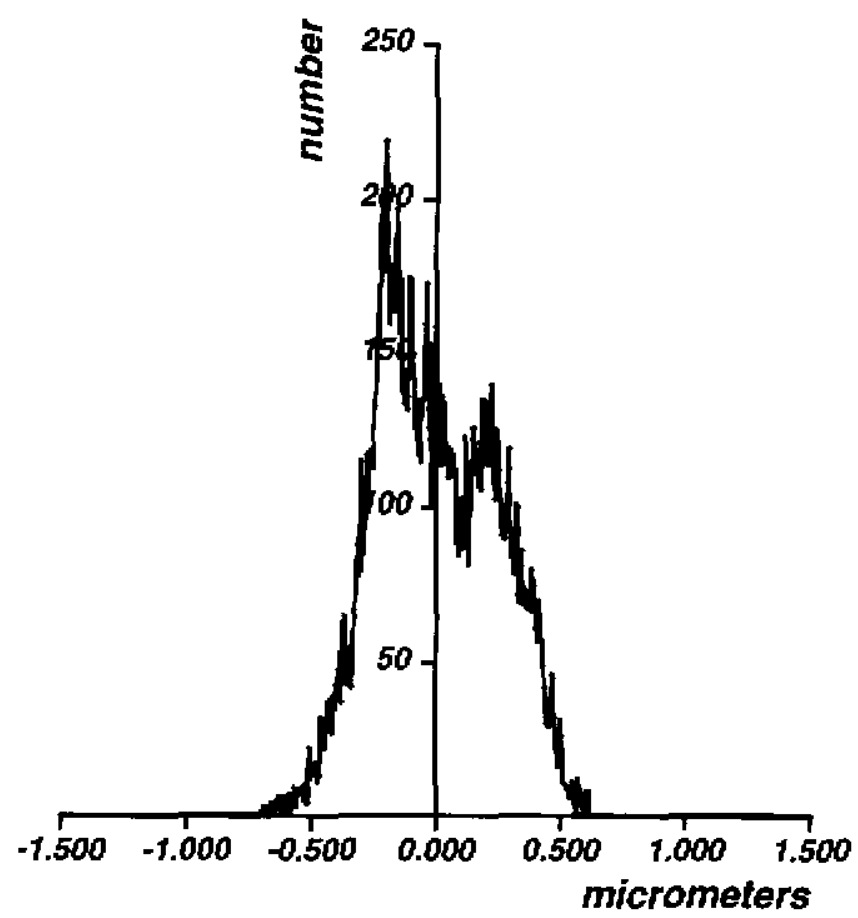

7.5 microinches sample

Figure 23: Number of occurrences of each height value

tribution[10]. We therefore model these histograms as binomial distributions, and find the values of the mean height 


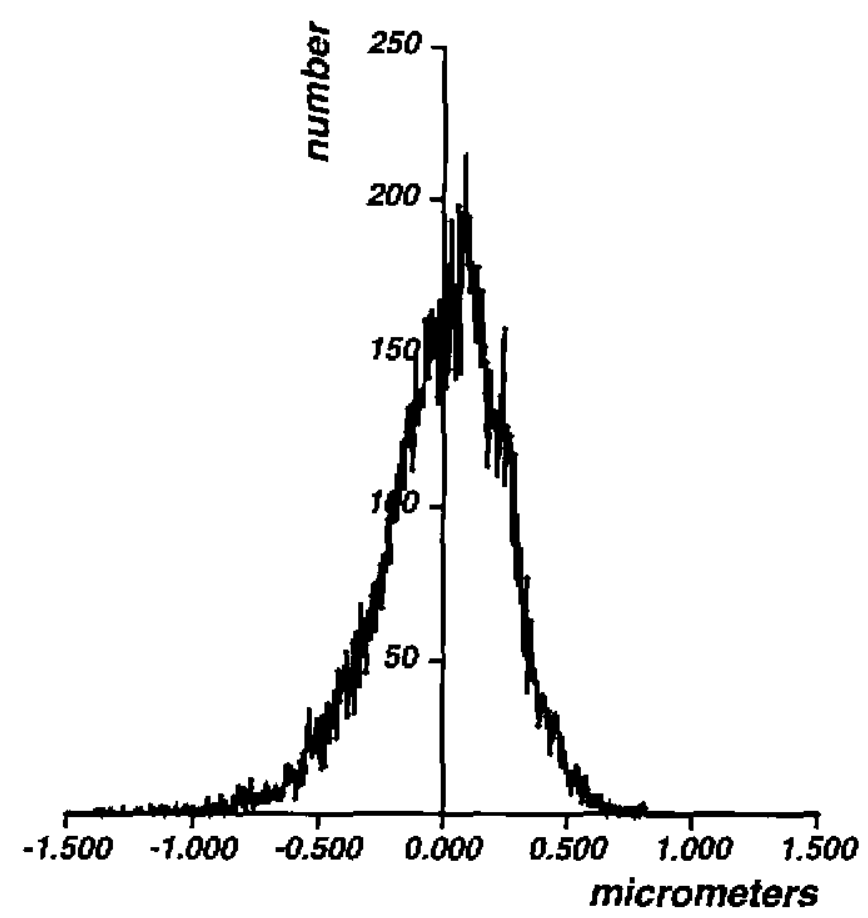

9.5 microinches sample

Figure 24: Number of occurrences of each height value

and roughness which give the best tit to the data. These values appear in Table 6. Notice that the mean values are not

\section{Mean height and roughness parameter}

sample

mean height (micrometers)

$\beta$

1.5 microinches

$-0.0025$

0.0588

3.0 microinches

$-0.0025$

0.104

4.5 microinches

$-0.0075$

0.216

7.5 microinches

$-0.0025$

0.351

9.5 microinches

$-0.0175$

0.443

Table 6 
zero, as they should be, and that the values of the roughness parameter are not the same as those shown in Table 3 . This is an indication that the surfaces do not follow a Gaussian height distribution. We next perform chi squared testing to determine if the fit is justified. The results of this procedure show that the surfaces actually are not Gaussian. This could be the cause for some of the error in the calculation of the $\mathrm{mms}$ roughness by optical means. The surfaces are close enough to having a Gaussian height distribution, however, that the procedure gives reasonable estimations of the roughness.

\section{Other roughness models}

We have now seen that it is possible to model certain rough surfaces with the one-dimensional Gaussian height FADF and to obtain roughness values in this way, but we have also seen that this model is often inadequate for real surfaces. We may rightfully ask if there are other roughness models which we may use.

\subsection{Isotropic Gaussian height model}

Another one of the most common FADF's is the isotropic Gaussian height model. This function describes surfaces which have a Gaussian distribution of height yalues in all directions. This was also proposed and studied by Beckmann and Spizzichino; its limiting case is the distribution function of Torrance and Sparrow. It has the form[4]

$$
S\left(\theta_{f} \varphi_{f}\right)=\frac{e^{-\left(\frac{\left.\tan \theta_{f}\right)^{2}}{\beta}\right)^{2}}}{\beta^{2} \pi\left(\cos \theta_{f}\right)^{4}}
$$

Let us now find the reflected radiance from a surface patch with this FADF. As before, we may use equation (6) to determine this value, although we now integrate over both angular variables. The reflected radiance will therefore be given by

$$
L_{r}=\int_{\varphi_{g 0} \theta_{j 0}}^{\varphi_{f 1} \theta_{f 1}} \frac{l\left(\cos \theta_{f} \cos \theta_{r}+\sin \theta_{f} \sin \theta_{r} \cos \left(\varphi_{f}-\varphi_{r}\right)\right) e^{-\left(\frac{\tan \theta_{f}}{\beta}\right)^{2}} \sin \theta_{f} d \theta_{f} d \varphi_{f}}{\beta \sqrt{\pi}\left(\cos \theta_{f}\right)^{4} \cos \theta_{r}}
$$

where $\theta_{f 0}$ and $\theta_{f 1}$ are now both functions of $\varphi_{f}$. The integral over $\theta_{f}$ of the first term in equation (20) is

$$
\int \frac{-e^{-\left(\tan \theta_{f}\right)^{2}}}{2} d \varphi_{f}
$$

We may integrate the second term by parts over $\theta_{f}$ to obtain

$$
\int \frac{l}{2}\left(\tan \theta_{f} e^{-\left(\tan \theta_{f}\right)^{2}}+e r f\left(\tan \theta_{f}\right)\right) d \varphi_{f}
$$

so that

$$
L_{r}=\left.\int_{\varphi_{f 1}}^{\varphi_{f 1}} \frac{l}{2}\left(-e^{-\left(\tan \theta_{j}\right)^{2}}+\tan \theta_{r} \cos \left(\varphi_{f}-\varphi_{r}\right)\left(\tan \theta_{f} e^{-\left(\tan \theta_{f}\right)^{2}}+\operatorname{erf}\left(\tan \theta_{f}\right)\right)\right)\right|_{\theta_{f l}} ^{\theta_{f 1}} d \varphi_{f}
$$


where $\theta_{f o}$ and $\varphi_{f 1}$ are functions of $\varphi_{f}$. We will evaluate this integral numerically. We are currently using this equation to model sandblasted surfaces, and are testing the appropriateness of the model.

\subsection{General one-dimensional FADF}

The one-dimensional Gaussian height model and the isotropic Gaussian height model cannot describe all interesting surfaces, despite the hopes of some machine vision researchers. Consider, for example, Figure 25, which shows a typ-

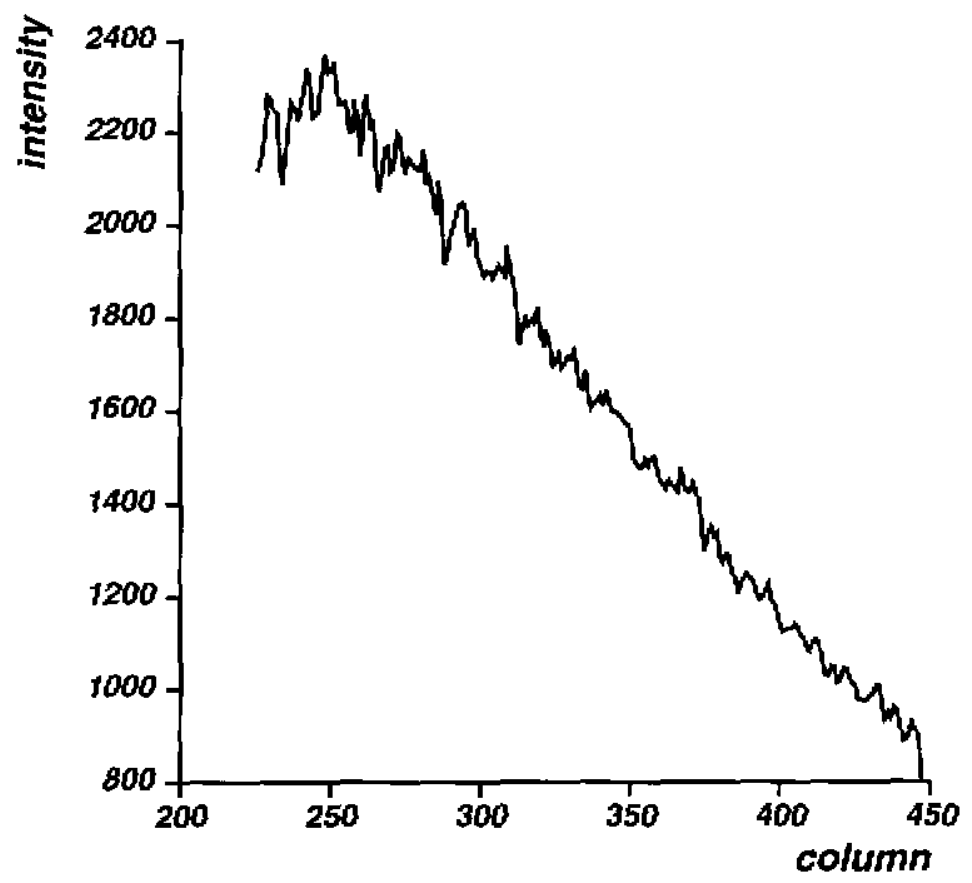

Figure 25: Edge profile for a ground surface

ical scanline from a metal plate which was finished by grinding. Notice that the edge profile is almost linear. The facet area distribution function for this surface is obviously very different from that proposed by Beckmann and Spizzichino, and our current method would therefore be of questionable value. It might be possible, however, to obtain information about the roughness of surfaces such as this one, which are not fit well by the Gaussian height models.

The method for estimating roughness which we have analyzed in this paper finds a single roughness parameter for the surface from all the points in the edge profile. This is a wise plan of attack, since it is an overdetermined system, and is therefore fairly robust with respect to noise. We have noted, however, that for one-dimensional FADF's with certain source and object configurations, the derivative of the edge profile is closely linked to the distribution function. Therefore, rather than propose a model of the rough surface and find one roughness measure as we do now, we hope to be able to obtain the shape of the FADF from the derivative of the edge profile. Because there are more degrees of freedom in the output of this method than there are in the output of our present method, we expect the derivative method to be more sensitive to noise. The method will also be more sensitive to noise because the derivative operator acts as a high pass filter. Nonetheless, our initial experiments at obtaining one-dimensional FADF's from reflected edges are promising, and it may soon develop into a tool applicable to a general vision system. Figure 26 to Figure 30 
show the FADF's found with this method for the five milled stainless steel plates.

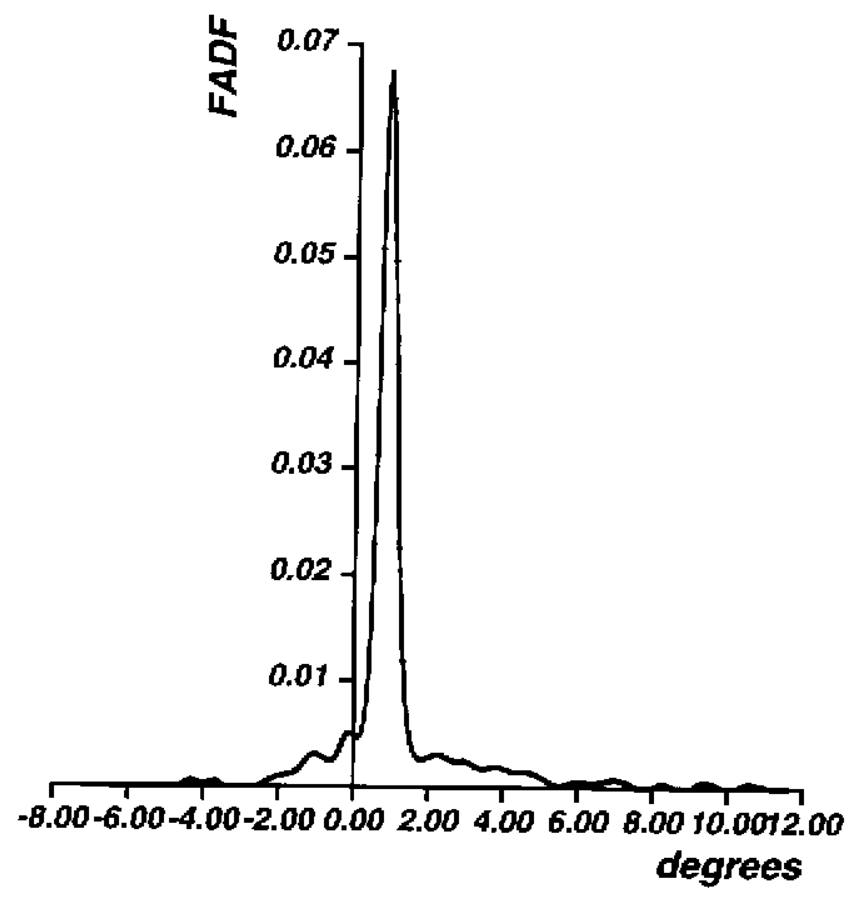

FADF for 1.5 microinches sample

Figure 26

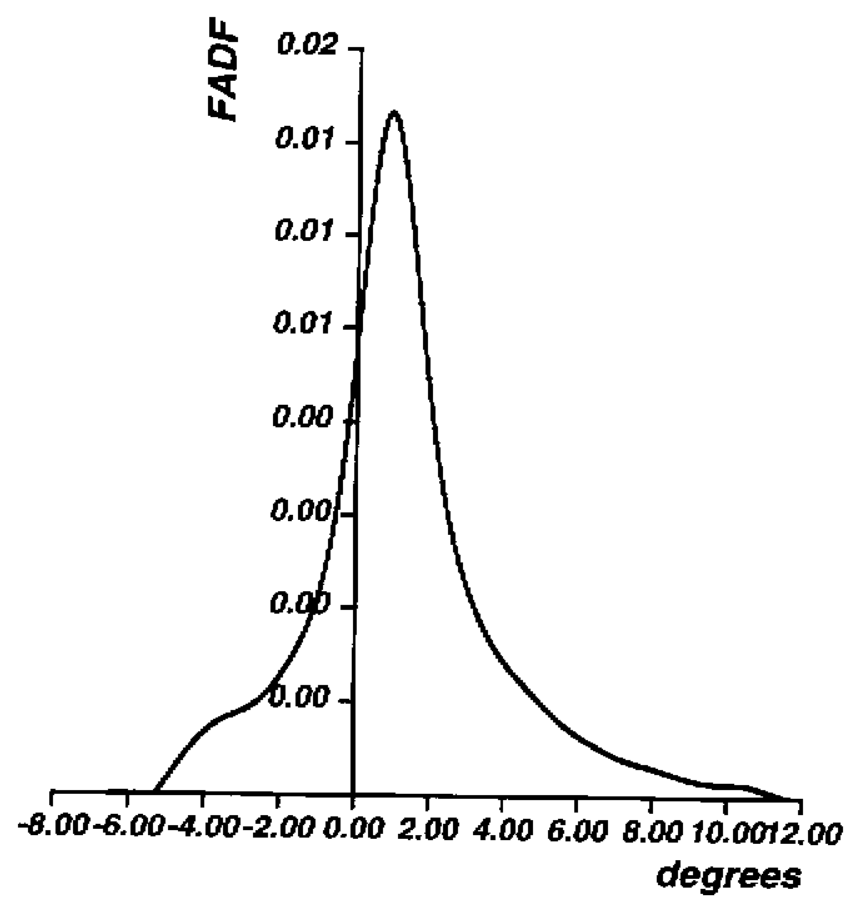

FADF for 3.0 microinches sample

Figure 27 


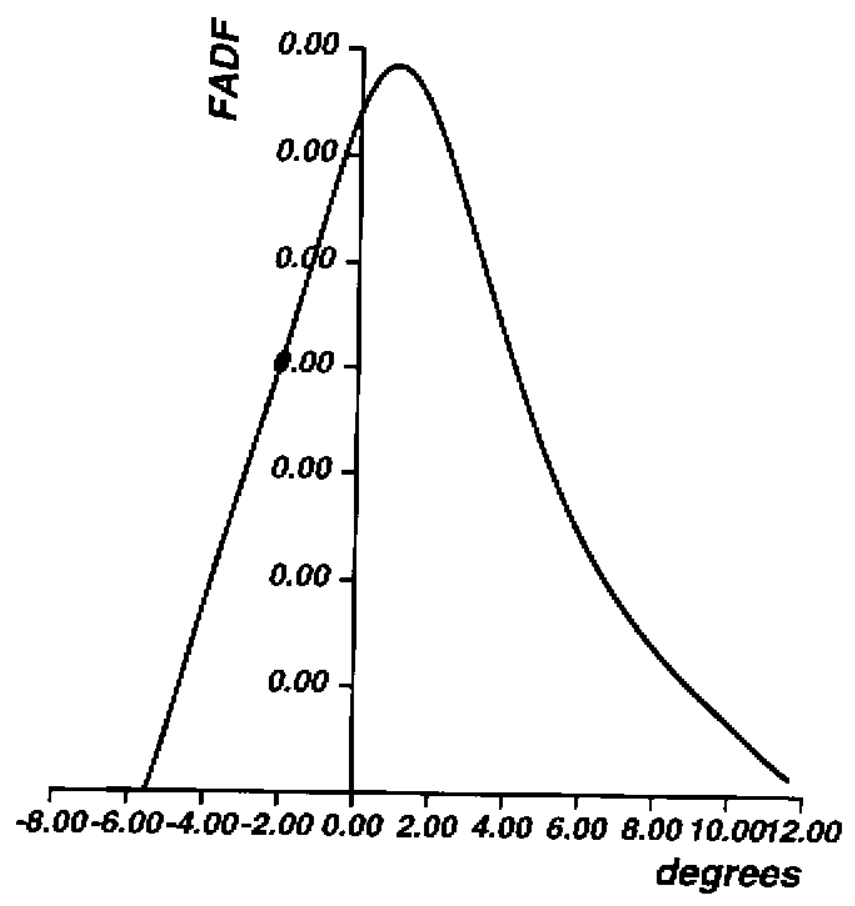

FADF for 4.5 microinches sample

Figure 28

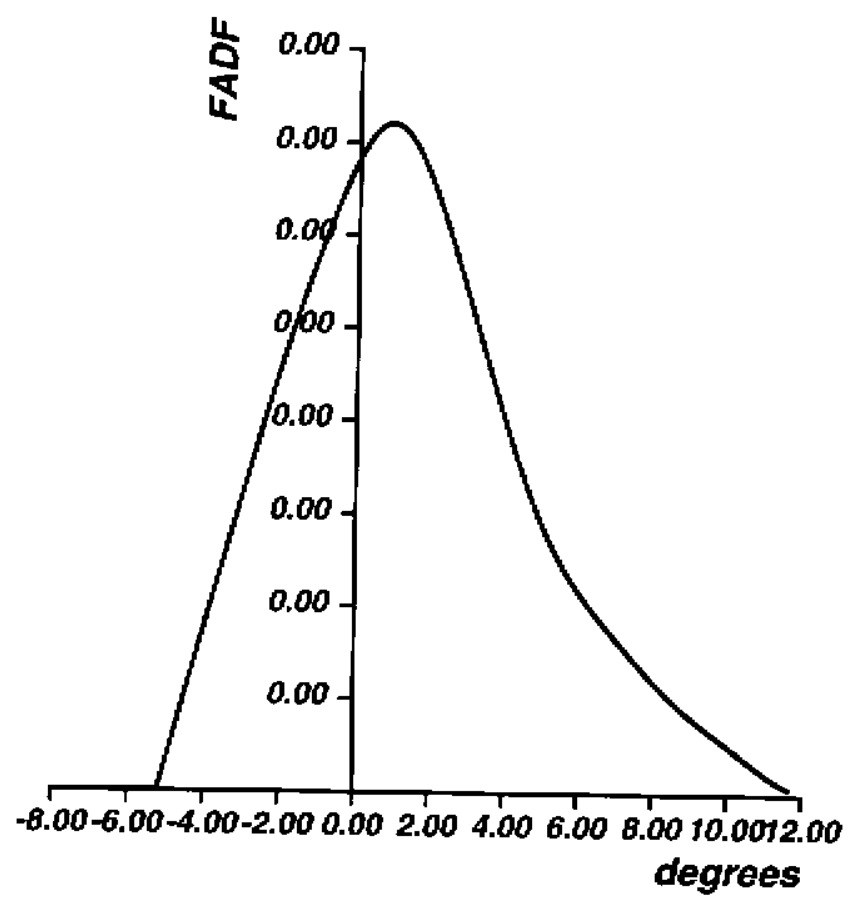

FADF for 7.5 microinches sample

Figure 29 


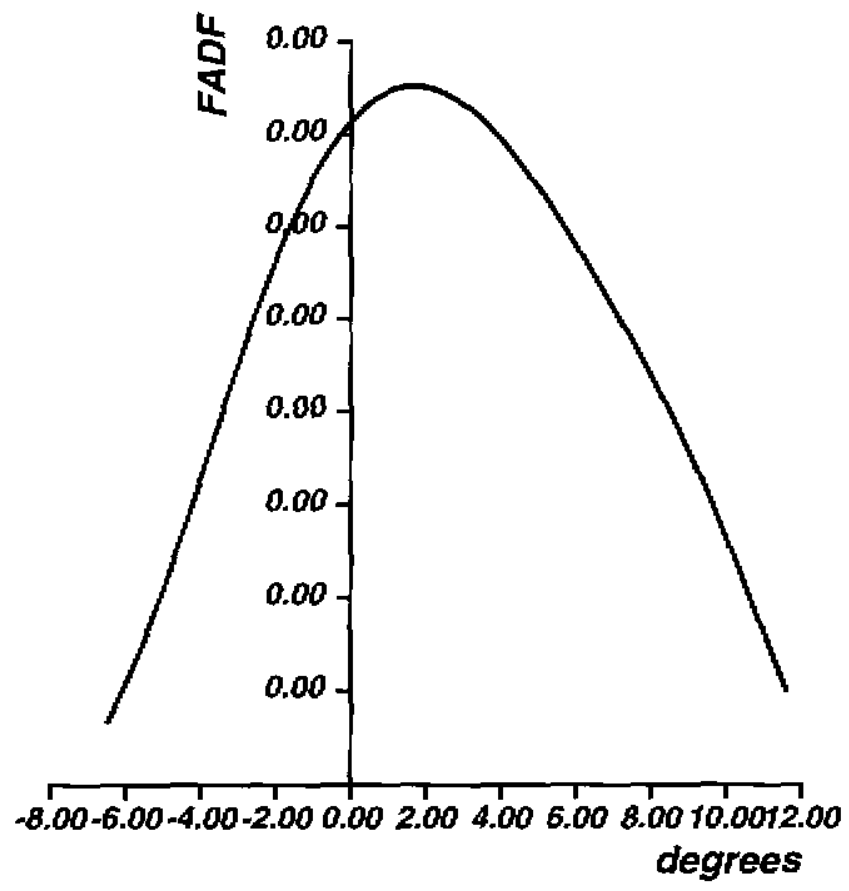

FADF for 9.5 microinches sample

Figure 30

\section{Implications for General Vision}

The analysis presented above shows that our method of roughness estimation poses no threat of supplanting curtent methods of roughness measurement. It could, however, prove useful in general purpose vision systems. The ability to analyze the reflections of step edges can be very useful in artificial environments, where edges are ubiquitous, and control over the environment is limited. Additionally, the theory of edge detection and localization is well developed, so the initial steps in such a vision system would be based on proven methods.

Such a system would begin by identifying the regions in an image appropriate for this analysis. It would then look for the reflected images of edges in each region. The sharpest reflected edges in the image would probably come from step edges in the environment, and therefore, it would focus the most attention on these reflected edges. These would also have the greatest high frequency content, and therefore, this search would be facilitated by a frequency space representation of the image, such as the spectrogram or local Fourier transform. Finally, the system would use the previously derived methods to estimate the roughness of the surface in the given region.

In order to construct a system such as this, however, there are several shortcomings which must be noted. The most obvious is the assumption of a known source position. The position of the source, i.e., the values of $\theta_{f 0}$ and $\theta_{j 1}$ for every position on the object, influences the reflected step edge profile drastically. Figure 31 shows the reflected edge profiles of the same surface with two different source positions. The two curves appear quite different and could be mistaken as coming from surfaces with different roughness values. Conversely, it is possible to generate similar reflected edge profiles for surfaces with quite different roughness values. Figure 32 shows a simulation of the 


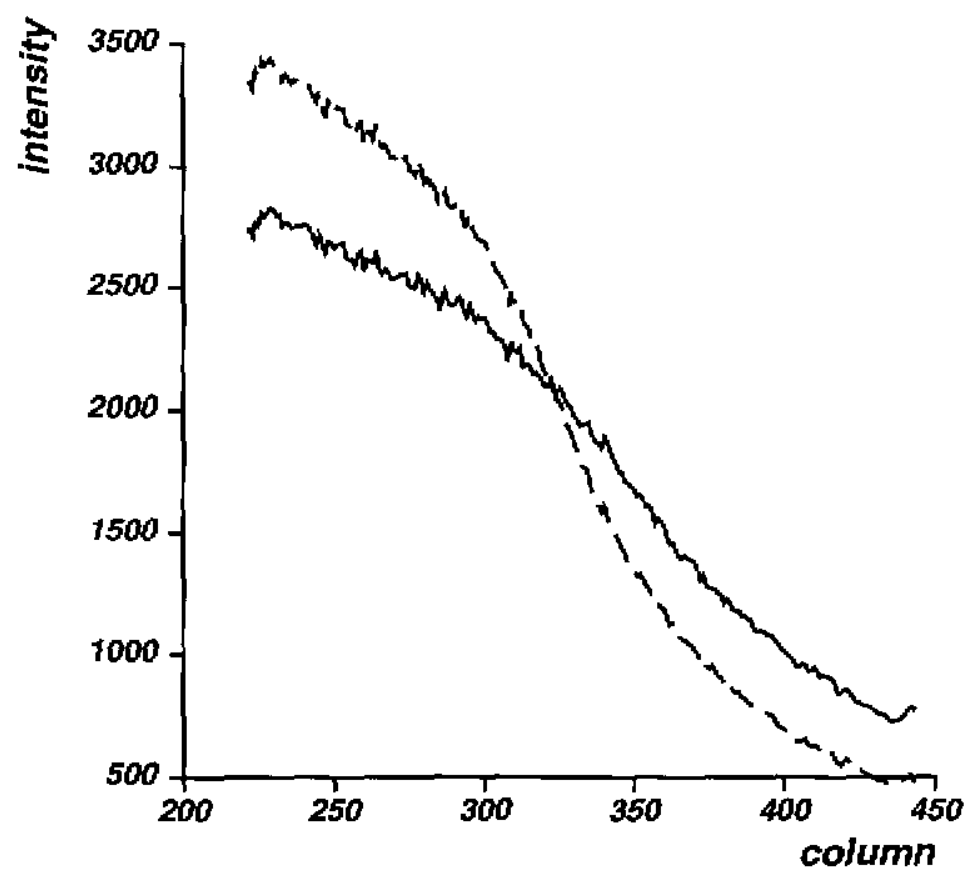

Figure 31: Reflected edge profiles from the same surface but with two different source positions

reflected edges of two surfaces with roughness parameters $\beta=0.1$ and $\beta=0.01$. Because of the different source

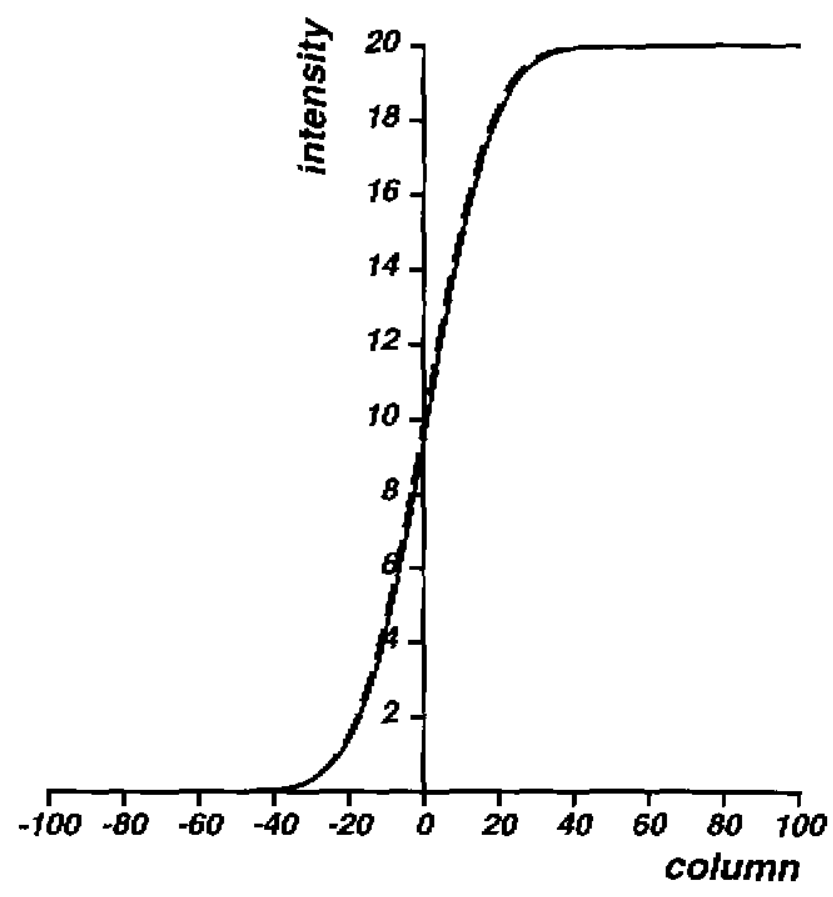

Figure 32: Two simulated edge profiles. The roughness values and source positions are different for each.

positions for each surface, the curves look very similar. In real data, noise could easily erase any differences between these curves. If we consider equation (15), we find part of the problem. In many cases, the error function terms in this equation dominate the other terms, and thus, the curve will be invariant for all cases in which 
$\operatorname{erf}\left(\frac{\tan \theta_{f 1}}{\beta}\right)-\operatorname{erf}\left(\frac{\tan \theta_{f 0}}{\beta}\right)$ equals a constant. Although we may not be able to determine the surface roughness in such cases, we may be able to determine the value of $\operatorname{erf}\left(\frac{\tan \theta_{f 1}}{\beta}\right)-\operatorname{erf}\left(\frac{\tan \theta_{f 0}}{\beta}\right)$ or $\frac{\tan \theta_{f 1}}{\beta}$ and $\frac{\tan \theta_{f 0}}{\beta}$, however. Another possible method to determine roughness without a known source position is to estimate the source position at the same time as estimating the roughness. It is also possible that frequency space and wayelet formulations might not need the knowledge of a source position.

Note that by studying the derivative of the edge profile, we obtain information only in the direction perpendicular to the edge, and this limits the knowledge we can gain about two-dimensional distribution functions. If there are multiple edges in an image, however, we may obtain information about the directional character of the roughness. Figure 33 and Figure 34 show the scanlines from the same surface perpendicular to two different edges. The directional

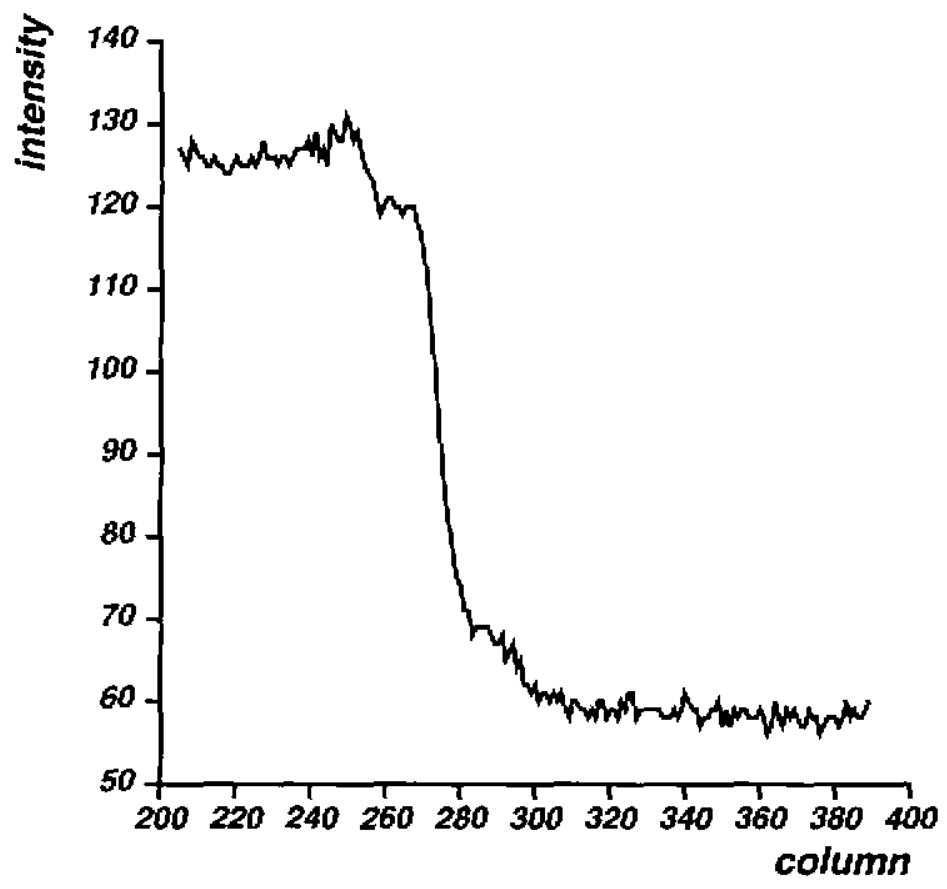

Figure 33: Profile of a step edge reflected in a rough surface

character of the roughness is obvious from these graphs. By following these lines of reasoning, we hope to improve our methods of roughness estimation to make them part of a general machine vision system.

\section{Conclusion}

We have shown that we may estimate the roughness of metal surfaces by studying the reflection of step edges. The method orders the surfaces the same as would a human observer, and this ordering appears related to the rms roughness of the surfaces. The values obtained are similar to those determined by other computer vision researchers, but are very different from those obtained by other means. Therefore, the method is probably impractical for precise measurements or quality control; it would, however, be useful for a general purpose vision system. In order to achieve this goal, we must remove our assumption of the known light source position. We do this in future work. We also discussed possible solution methods for surfaces with non-Gaussian height distributions, and the application of such methods to a general vision system. 


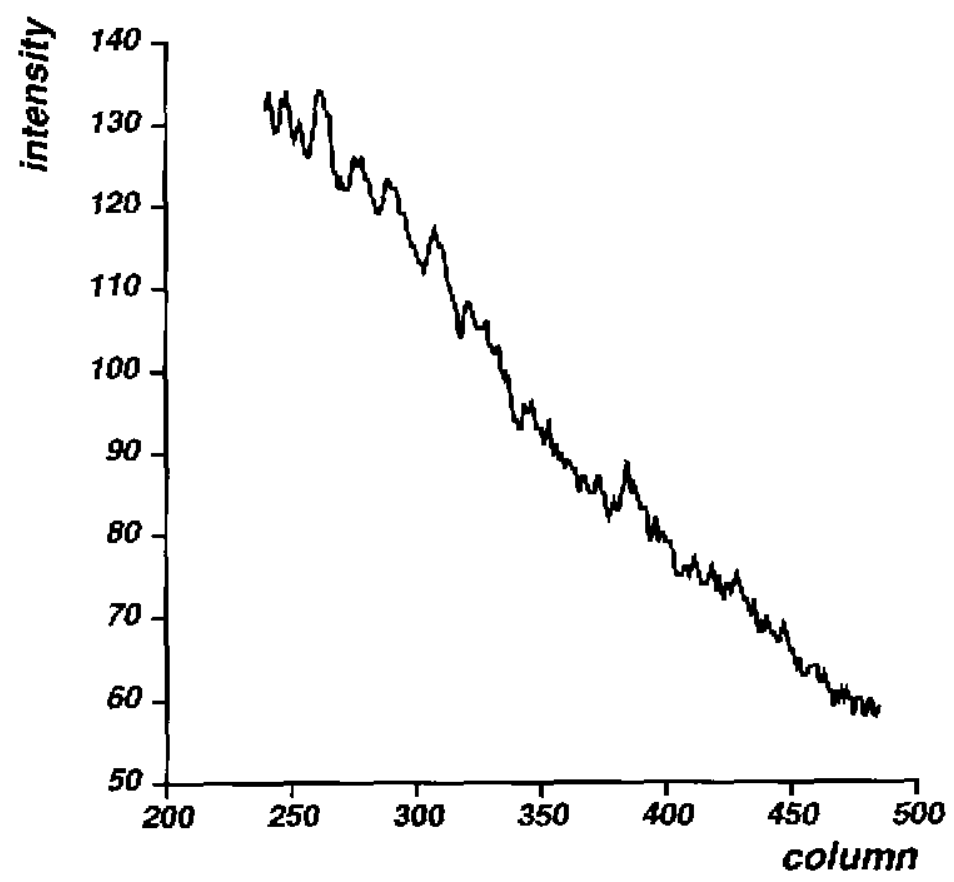

Figure 34: Profile of a step edge reflected in the same rough surface, but with the perpendicular orientation

\section{Bibliography}

1. Beckmann, Petr, and Spizzichino, Andre, The Reffection of Electromagnetic Waves from Rough Surfaces, Norwood, MA., Artech House, 1987.

2. Bennett, J. M., and Mattson, L., Introduction to Surface Roughness and Scattering, (Optical Society of America, Washington, D.C., 1989).

3. Blinn, J. F., Models of Light Reflection for Computer Synthesized Pictures, Computer Graphics, vol. 11, no. 2, 1977, pp. $192-198$.

4. Cook, R. L., and Torrance, K. E., A Reflectance Model for Computer Graphics, ACM Transactions on Graphics, vol. 1, no.1, January, 1982, pp. 7 - 24.

5 Ilealey, Glenn, and Binford, Thomas O., Local Shape from Specularity, Computer Vision, Graphics, and Image Processing, vol. 42,1988 , pp. $62-86$.

6. Jaggard, Dwight $\mathrm{I}_{\text {, }}$ and Sun, Xiaoguang, Scattering from Fractally Corrugated Surfaces, J. Opt. Soc. Am. A, vol. 7, no. 6, June 1990. pp. $1131-1139$.

7. Nayar, Shree K., Ikeuchi, Katsushi, and Kanade, Takeo, Determining Shape and Reflectance of Hybrid Surfaces by Photometric Sampling, IEEE Transactions on Robotics and Automation, vol. 6, no. 4, August, 1990.

8. Nayar, Shree K. Ikeuchi, Katsushi, and Kanade, Takeo, Surface Reflection: Physical and Geometrical Perspectives, CMU-RI-TR-89-7, March, 1989.

9. Novak, Carol, Estimating Scene Properties by Analyzing Color Histograns with Physics-Based Models, Ph.D. thesis, Carnegie Mellon University, 1992. 
10. Stone, Ronald, and Shafer, Steven, The Determination of Surface Roughness from Reflected Step Edges, Technical Report (in progress), Carnegie Mellon University, 1993, Appendix 1.

10. Stover, John C.. Optical Scattering Measurement and Analysis, New York, McGraw-Hill, Inc., 1990.

11. Torrance, K. E., and Sparrow, E. M., Theory for Off-Specular Reflection From Roughened Surfaces, Journal of the Optical Society of America, vol. 57, no. 9, September, 1967.

\section{Acknowledgments}

We wish to thank Alcoa, Inc. for their generous loan of the milled stainless steel plates.

\section{Appendix}

We wish to determine if the surfaces actually exhibit a Gaussian distribution of height values. Therefore, for each surface, we made a histogram of the number of occurrences of each height value in the ten protiles for each surface. These histograms appeared in Figure 19 to Figure 25. As the number of measured points increases, these histograms should approximate a Gaussian distribution more and more closely. The true form of the histograms can be derived, however. We first consider the case of no measurement errors. Consider a discretely sampled rough surface with a Gaussian distribution of heights. The probability of a given sample being in the height range $h$ to $h+d h$ is

$$
P=\frac{e^{-\frac{(h-\mu)^{2}}{2 \sigma_{h}^{2}}}}{\sqrt{2 \pi} \sigma_{h}} d h
$$

where $\sigma_{h}$ is the standard deviation of the distribution. Now, if we have a scan of the surface with $S$ sample points, the probability that $n$ of them will be in the range from $h$ to $h+d h$ is given by the binomial distribution, or

$$
\text { probability }=\left(\begin{array}{l}
S \\
n
\end{array}\right) P^{n}(1-P)^{S-n}
$$

The mean and variance of the binomial distribution are known to be $S P$ and $S P(1-P)$, respectively.

If we now allow the existence of measurement errors, we must change the value of $P$. The probability that a measured point has value $h_{i}$ is, as before,

$$
p_{i}=\frac{e^{-\frac{\left(h_{i}-\mu\right)^{2}}{2 \sigma_{h}^{2}}}}{\sqrt{2 \pi} \sigma_{h}} d h_{i}
$$

The probability that the measuring instrument will record the value of this point as $h$ is

$$
q_{i}=\frac{e^{-\frac{\left(h-h_{i}\right)^{2}}{2 \sigma_{m}^{2}}}}{\sqrt{2 \pi} \sigma_{m}} d h
$$


where we have assumed that the measurement errors are Gaussian distributed with standard deviation $\sigma_{m}$. The probability that the point has value $h_{i}$, but is recorded as $h$ is then $p_{i} q_{i}$. We sum these probabilities over all possible values of $h_{i}$ to determine the probability of a point having measured value in the range from $h$ to $h+d h$. This probability is

$$
P=\sum p_{i} q_{i}=\sum\left(\frac{e^{-\frac{\left(h_{i}-\mu\right)^{2}}{2 \sigma_{h}^{2}}}}{\sqrt{2 \pi} \sigma_{h}} d h_{i}\right)\left(\frac{e^{-\frac{\left(h-h_{i}\right)^{2}}{2 \sigma_{m}^{2}}}}{\sqrt{2 \pi} \sigma_{m}} d h\right)
$$

If $\sigma_{m} * \sigma_{h}$, i.e. if the measurement errors are much smaller than the standard deviation of the height distribution, as is our case, the second term in the sum is appreciable only for a small range of values of $i$, and the first term is fairly constant over this range. Therefore, we may approximate $h_{i}$ in the first term by $h$, and factor out the first term to find

$$
P=\left(\frac { e ^ { - \frac { ( h - \mu ) ^ { 2 } } { 2 \sigma _ { h } ^ { 2 } } } } { \sqrt { 2 \pi } \sigma _ { h } } d h \left(\sum\left(\frac{e^{-\frac{\left(h-h_{i}\right)^{2}}{2 \sigma_{m}^{2}}}}{\sqrt{2 \pi} \sigma_{m}} d h\right)\right.\right.
$$

The sum is approximately equal to one, so that

$$
P \approx \frac{e^{-\frac{(h-\mu)^{2}}{2 \sigma_{h}^{2}}}}{\sqrt{2 \pi} \sigma_{h}} d h
$$

and we may use the results we derived for the case with no measurement errors. 